Related content

\section{Sequestering $\mathrm{CP}$ violation and GIM-violation with warped extra dimensions}

To cite this article: Clifford Cheung et al JHEP01(2008)069

View the article online for updates and enhancements.

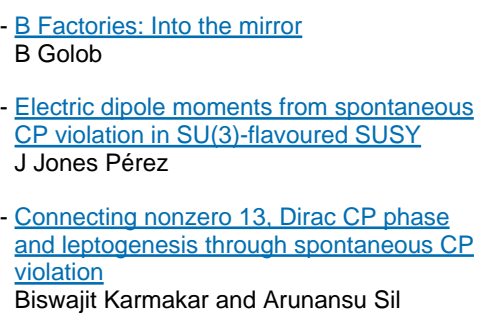

Biswajit Karmakar and Arunansu Sil

Recent citations

- Solving the strong CP problem with nonconventional CP

A. L. Cherchiglia and C. C. Nishi

- A flavorful factoring of the strong CP problem

Prateek Agrawal and Kiel Howe

Spontaneous CP violation and the strong

CP problem

Luca Vecchi 


\title{
Sequestering CP violation and GIM-violation with warped extra dimensions
}

\author{
Clifford Cheung, A. Liam Fitzpatrick and Lisa Randall \\ Jefferson Physical Laboratory, \\ Harvard University, Cambridge, MA 02138, U.S.A. \\ E-mail: cwcheung@fas.harvard.edu, fitzpatrick@physics.harvard.edu, \\ randall@physics.harvard.edu
}

Abstract: We propose a model of spontaneous $\mathrm{CP}$ violation to address the strong $\mathrm{CP}$ problem in warped extra dimensions that relies on sequestering flavor and $\mathrm{CP}$ violation. We assume that brane-localized Higgs Yukawa interactions respect a U(3) flavor symmetry that is broken only by bulk fermion mass and Yukawa terms. All CP violation arises from the vev of a CP-odd scalar field localized in the bulk. To suppress radiative corrections to $\bar{\theta}$, the doublet quarks in this model are localized on the IR brane. We calculate constraints from flavor-changing neutral currents (FCNCs), precision electroweak measurements, CKM unitarity, and the electric dipole moments in this model and predict $\bar{\theta}$ to be at least about $10^{-12}$

Keywords: Technicolor and Composite Models, CP violation, Quark Masses and SM

Parameters. 


\section{Contents}

1. Introduction 2

2. Flavor physics in higher-dimensional models 5

3. The model 7

3.1 Definition of the model 7

3.2 Zero mode wavefunctions 8

3.3 Approximate dependence of CKM angles on $t_{0}$

3.4 Specific values 11

3.5 Fermion wavefunction dependence on the bulk masses 12

4. Interactions in the KK reduced theory 13

4.1 Yukawa interactions

5. CP and flavor constraints

5.1 Contributions to $\Phi$ interactions from higher-dimensional operators 15

5.2 The EDM operator and $\bar{\theta}$ at one loop 16

$5.3 \quad \bar{\theta}$ at two loops from $W$ 's and higgses 17

$5.4 \bar{\theta}$ from flavor spurions 21

5.5 Unitarity of $V_{\mathrm{CKM}}$

5.6 Flavor-changing neutral currents 22

6. Conclusion 23

A. Bulk fermion wavefunctions 24

A.1 Wavefunctions without twisting 24

A.2 Wavefunctions with twisting 26

B. Analysis of flavor spurions in small $t_{0}$ limit 28

G. Precision electroweak constraints 33

C.1 Contributions from KK modes 34

D. Domain walls 36

E. KK fermion mass matrix 36 


\section{Introduction}

$\mathrm{CP}$ violation in the kaon system was first observed more than 40 years ago [1], and yet no evidence of $\mathrm{CP}$ violation in the strong interactions has since been observed. Whereas the weak phase is of order unity, the strong CP angle $\bar{\theta}$ is constrained to be $\lesssim 10^{-10}$.

The quarks get their masses in the Standard Model from two Yukawa matrices $Y_{u}, Y_{d}$ for the up-type quarks and down-type quarks, respectively, which induce a unitary CKM matrix when rotated into the mass eigenbasis:

$$
\begin{aligned}
\lambda_{u} & \rightarrow L_{u} \lambda_{u} R_{u}^{\dagger}=\mu_{u} / v \\
\lambda_{d} & \rightarrow L_{d} \lambda_{d} R_{d}^{\dagger}=\mu_{d} / v \\
V_{\mathrm{CKM}} & =L_{u} L_{d}^{\dagger}
\end{aligned}
$$

$V_{\mathrm{CKM}}$ contains a single phase that cannot be removed by vector-like rotations of the quarks. This phase accounts for all observed CP violation in the Standard Model. The rotation in equation (1.1) is generically chiral and thus shifts $\theta_{\mathrm{QCD}}$, but in such a way that the physically observable $\bar{\theta}$ remains invariant

$$
\bar{\theta}=\theta_{\mathrm{QCD}}-\arg \operatorname{det} \lambda_{u} \lambda_{d}
$$

The renormalizable operator $\theta_{\mathrm{QCD}} G \tilde{G}$ could in principle violate $\mathrm{CP}$ independently of the weak interactions. Without some mechanism for preventing strong $\mathrm{CP}$ violation, the cancellation between $\theta_{\mathrm{QCD}}$ and $\operatorname{argdet} \lambda_{u} \lambda_{d}$ appears extremely fine-tuned.

An additional difficulty with constraints on $\mathrm{CP}$ violation occurs in models where the flavor structure of the Standard Model is explained by UV physics. Since flavor and CP in the Standard Model have the same origin (the Yukawa matrices), any solution to the flavor problem is likely to also introduce new sources of CP violation (CPV). Integrating out heavy fields generically induces dimension- 5 operators that contribute to the neutron electric dipole moment (EDM), independently of $\bar{\theta}$. Axion models, which address only $\bar{\theta}$ have nothing to say about these additional contributions. Of course the order of magnitude involved is far less severe, but nonetheless these additional contributions to EDMs generally require additional model-building constraints.

The approach we take addresses these issues and falls in the class of solutions referred to as spontaneous CP violation. The idea is that CP is a valid symmetry in the UV, but is broken spontaneously in such a way that phases enter $V_{\mathrm{CKM}}$ but not $\arg \operatorname{det} \lambda_{u} \lambda_{d}$.

In this paper we consider a theory of spontaneous CPV embedded in RS. We utilize in a warped scenario the extra dimensional mechanism of [12], which "sequesters" the source of CPV from operators that could directly transmit it to $\bar{\theta}$. Thus, we are able to address the strong $\mathrm{CP}$ problem even with new KK modes near the electroweak scale, though electroweak constraints push the KK masses to be $\gtrsim 15-18 \mathrm{TeV}$. This is low from the point of view of models addressing the strong CP problem, though unfortunately it is fairly high in terms of addressing the gauge hierarchy problem. However we show that embedding a solution to the strong CP problem based on spontaneous CPV inside RS allows us to explain mass scales and further suppress some potentially dangerous $\mathrm{CP}$ and 
flavor violating contributions, thereby allowing us to address both the gauge hierarchy and fermion mass hierarchy problems.

Furthermore, sequestering CPV relaxes constraints on the KK scale of RS from neutron electric dipole moment (EDM) measurements. We will show that if CPV is sourced in the bulk and transmitted through the fermion wavefunctions, then the flavor violation and $\mathrm{CP}$ violation very efficiently "washes out" of the excited modes but not the zero modes. This is to be contrasted with "anarchic" models of flavor in RS, where the CPV comes from Higgs Yukawas with $\mathcal{O}(1)$ entries and phases, and thus is equally present at every KK level. We show that our model suppresses EDM operators radiatively generated from integrating out KK modes.

As we will explain in more detail throughout the paper, the suppression of flavor and $\mathrm{CP}$ violation in our model exploits four ingredients that are natural in the context of extra dimensions:

1. AdS spacetime isometries in the bulk. The $\mathrm{SO}(4,2)$ symmetry of AdS greatly restricts the possible couplings of bulk fermions a bulk scalar field $\Phi$, analogous to the restrictions imposed by 5D Lorentz symmetry in flat-space [12]. We assume CP is spontaneously broken when $\Phi$ gets a vev. This generates a hermitian bulk mass matrix $\mathcal{M}$ for the fermions as with the mechanism of [12]. The zero mode wavefunctions $F^{(0)}(\phi)$ at a position $\phi$ in the bulk then take the form

$$
F^{(0)} \propto \mathcal{P} \exp \left(\int^{\phi} \mathcal{M}\left(\phi^{\prime}\right) d \phi^{\prime}\right)
$$

where $\mathcal{P}$ denotes path ordering with respect to $\phi$. This has real determinant since $\mathcal{M}$ is Hermitian. Taking $\lambda_{u, d}$ to be the brane Yukawas, which are real-valued by $\mathrm{CP}$, one finds that $Y_{u, d}=F_{q}^{(0)} \lambda_{u, d} F_{u, d}^{(0)}$. Both have real determinant, and $\bar{\theta}$ vanishes at tree-level.

2. Sequestering of the CP violating scalar field from the boundaries and the local Yukawa interactions. Ingredient (1) enforces that $\mathrm{CP}$ violation in the bulk that is transmitted through the fermion wavefunctions will not induce $\bar{\theta}$ at tree level. However, if the source of CPV has non-negligible overlap with the TeV brane, then it can enter the Yukawas through the direct coupling $\Phi_{i j}\left(\bar{Q}_{L}\right)_{i} H\left(U_{R}\right)_{j}$, which contributes order one strong CP phases once $\Phi$ gets a vev. $\bar{\theta}$ would not be protected from these contributions, and thus it is crucial that the source of CPV be geographically sequestered from the Higgs Yukawas. Furthermore, the AdS isometries are explicitly broken at the boundaries of RS, and the coupling of $\Phi$ to the fermions is not protected there. We therefore assume that $\langle\Phi\rangle$ is localized somewhere in the bulk, away from the boundary branes. We will see that the farther we localize $\Phi$ in the UV, the less its effect will be on the KK fermion wavefunctions, so CP violation is even more effectively sequestered than one might naively assume.

For simplicity, we sequester $\Phi$ by localizing it to a third brane in the warped bulk. It is possible to imagine other equally viable profiles for $\Phi$. It will be important 
that the effect of $\Phi$ washes out much more efficiently of the IR-localized KK mode wavefunctions than in those of the UV-localized zero modes so that there is a limit where the KK mode wavefunctions are diagonal in flavor indices (up to negligible corrections) and all CP violation enters the KK-reduced theory only through the zero mode wavefunctions.

3. A large flavor symmetry. At tree-level, the only aspect of the flavor symmetry that is necessary for $\bar{\theta}$ suppression is that all the up-type quarks get their masses at the same place in the bulk, and similarly for the down-type quarks. This is generically a feature of RSI models, where the Higgs vev must be localized near the TeV brane in order to solve the hierarchy problem. To address loop corrections, we assume that the flavor group $\mathrm{U}(3)_{Q} \times \mathrm{U}(3)_{U} \times \mathrm{U}(3)_{D}$ breaks to a diagonal $\mathrm{U}(3)$ through the Higgs Yukawas, and further down to the $\mathrm{U}(1)^{3}$ subgroup by constant bulk fermion masses, and finally broken completely by a CPV source in the bulk.

We further assume that the bulk masses for the up-type singlets and down-type singlets approximately commute, so that they are diagonal in approximately the same basis. ${ }^{1}$ In this case, the bulk masses leave invariant an approximate $\mathrm{U}(1)^{3}$ for each generation. It is not necessary to make any further assumption about the bulk mass matrices aligning with the brane Yukawas.

4. Doublets confined to the IR brane. The doublets are confined to the IR brane. Consequently, the only states charged under $\mathrm{SU}(2)_{L}$ are zero modes. This implies that all fermion interactions with charged currents are controlled by a single CKM matrix $V_{\text {CKM }}$ that is a generalization of the Standard Model version. In the Standard Model, the CKM matrix is unitary since all of the left-handed fields couple to the $W$ bosons with equal strength. However, in $5 \mathrm{~d}$ models, there are left-handed fields, namely the left-handed KK excitations of the electroweak singlets, which do not couple to $W$ bosons. Consequently, $V_{\mathrm{CKM}}$ is of the form

$$
V_{\mathrm{CKM}}=L_{u} P_{0} L_{d}^{\dagger}
$$

where $P_{0}$ is a projection onto the left-handed zero modes and $L_{u, d}$ are diagonalization matrices that mix zero and KK modes.

With the doublets to be confined to the IR brane, all CP and flavor violation appears through the singlet wavefunctions. In particular, the singlet zero mode wavefunctions are responsible for generating small Standard Model CKM angles. When the CPV source is located sufficiently far in the UV, the CKM angles appear generically as fractional powers of ratios of fermion masses, and thus are generated naturally.

We will see that a consequence of these assumptions is that up to $O\left(v / M_{\mathrm{KK}}\right)^{2}$, we have an approximate GIM mechanism. Our basic outline for this paper is as follows. In section 2 , we review flavor in warped extra-dimensional models and describe some differences in

\footnotetext{
${ }^{1}$ This could be achieved for instance by coupling together the fields responsible for generating their bulk masses so that their potential is minimized when they align.
} 
our model. We present our model and some of its features in section 3. In sections 4 we discuss the KK reduced theory and its interactions. In section 5, we calculate the $\mathrm{CP}$ and flavor constraints, and in section 6 we conclude.

\section{Flavor physics in higher-dimensional models}

Because the spontaneous breaking of $\mathrm{CP}$ is intimately connected with the breaking of flavor symmetries in our model, we give a brief aside about flavor physics in higher-dimensional models and RS in particular. The models we compare all have an extra dimension bounded by branes. The original RS model had the entirety of the SM localized on the IR brane. Confinement of the SM fields to the IR brane was assumed to happen through some unspecified dynamics whose effects on flavor-changing, etc., were impossible to calculate. Putting the SM gauge fields in the bulk (first considered in [8, 9]) allowed the possibility for gauge coupling unification [16-20], but it had calculable corrections to precision electroweak observables that raised the scale of KK gauge bosons to $23 \mathrm{TeV}$. Putting all the fermions in the bulk drastically weakened such constraints, and further allowed for the warping of fermion wavefunctions to explain the hierarchy in their masses [10, 11, 25, 22] while the brane Yukawas and masses were order $\mathcal{O}(1)$ and anarchic. In these "anarchic" models, the small zero mode masses and CKM angles arise from the hierarchy in fermion wavefunctions on the $\mathrm{TeV}$ brane, as follows. The Yukawa matrices in the KK reduced theory are $Y_{u, d}=F_{Q}^{(0)} \lambda_{u, d} F_{u, d}^{(0)}$, where $\lambda_{u, d}$ are the 5 d brane Yukawa matrices with $\mathcal{O}(1)$ values and phases in every entry and $F_{Q, u, d}^{(0)}$ are matrices of the zero mode wavefunctions at the IR brane. The zero mode masses then are dynamically generated as

$$
m_{u, d} \approx v F_{Q}^{(0)} F_{u, d}^{(0)}=v \sqrt{\frac{1+2 \nu_{Q}}{1-e^{-\left(1+2 \nu_{Q}\right) k \pi r_{c}}}} \sqrt{\frac{1+2 \nu_{u, d}}{1-e^{-\left(1+2 \nu_{u, d}\right) k \pi r_{c}}}}
$$

where $\nu_{Q, u, d}$ are the bulk masses in units of the AdS curvature scale $k$, and we have used the zero mode wavefunctions $F^{(0)}=\sqrt{(1+2 \nu) /\left(1-e^{\left.-(1+2 \nu) k \pi r_{c}\right)}\right.}$. Therefore, a modest spread in the values of $\nu$ for the different fermions gives rise to an exponential hierarchy in the masses. Furthermore, the CKM angles are controlled by the bulk masses for the doublets, as follows. The CKM matrix diagonalizes $Y_{u, d} Y_{u, d}^{\dagger}=F_{Q}^{(0)} \lambda_{u, d} F_{u, d}^{(0) 2} \lambda_{u, d}^{\dagger} F_{Q}^{(0)}$, and thus the CKM angles are approximately

$$
\theta_{i j} \approx \frac{F_{Q i}}{F_{Q j}}
$$

Constraints on the $T$ parameter from bulk SU(2) gauge boson contributions were further reduced in models in which global custodial $\mathrm{SU}(2)$ was extended to a gauge symmetry in the bulk. Potential problems with RS models of flavor are the $Z$ to $b \bar{b}$ coupling, which is modified because $b_{L}$ cannot be localized too far away from the IR brane, where KK modes of bulk gauge fields are concentrated, as well as large $\epsilon_{K}$ and the electric dipole moment of the neutron. These latter constraints are particularly bad because of the parity symmetry in the bulk, which means that both left and right-handed fields couple to the $W$ gauge boson KK modes. These constraints are quite severe [34, 35]. 


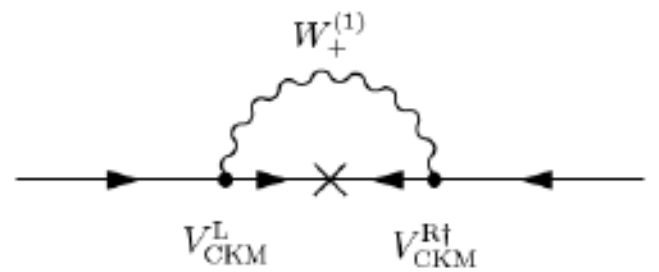

Figure 1: A one-loop contribution to $\bar{\theta}$ in the case where both the doublets and the singlets are in the bulk. We therefore take the doublets to be localized on the brane.

As in any model of flavor, one can also try to address the strong CP problem in these models. The first proposal to use twisting to solve the strong CP problem was made in [12], in a flat extra dimension. There, $\mathrm{CP}$ violation occurred because up and down type quarks were located at opposite ends of the fifth dimension so that CPV couldn't be eliminated from both simultaneously. However, RS requires that all Yukawas be on the IR brane, so it is necessary to generate different phases for the up and down type right handed fields, which can be done by placing them both in the bulk rather than on branes. Because of the strong EDM constraint, we do not allow left handed Standard Model fermions in the bulk but instead sequester them on the IR brane. The reason is that bulk doublets have a vector-like tower of KK modes, so that there exist right-handed fields charged under $\mathrm{SU}(2)_{L}$. Then, there is not only a CKM matrix for the left-handed fields, but an additional CKM matrix for the right-handed fields as well, and $\bar{\theta}$ would be renormalized at one-loop by the diagram in figure 1 . We thus consider brane-localized doublet fermions only.

However, as mentioned, models with light fermions localized on the $\mathrm{TeV}$ brane face very restrictive constraints from precision electroweak measurements, since the KK gluon wavefunctions are enhanced by a volume factor in the IR. Our strongest constraints will be from these measurements, which puts the scale of KK gauge bosons in our model at about $15-18 \mathrm{TeV}$. Similar though weaker constraints come from flavor and unitarity of the observed CKM matrix for the zero mode quarks.

It is nonetheless worth noting the interesting flavor physics of this model with bulk right handed fermions but IR-localized left-handed fermions. We will assume a U(1) ${ }^{3}$ symmetry is preserved by the bulk masses which is broken only by a localized CPV field in the bulk coupling to the bulk singlets through Yukawa couplings. Unlike in "anarchic" models, the brane Yukawa matrices in our model are flavor universal and thus the CKM matrix arises fairly naturally from the different values of the right-handed fermion wave functions, even though their bulk masses are similar and the vev of the CPV field is quite generic. More precisely, we will show that in our setup the Yukawas are given by

$$
Y_{u, d} \approx F_{u, d}^{(0)}=\left(\frac{1+2 \nu_{u, d}}{1-e^{\left(1+2 \nu_{u, d}\right) k \pi r_{c}}}\right)^{1-\frac{\phi_{0}}{\pi}} e^{k r_{c} g_{u, d} \Phi}\left(\frac{1+2 \nu_{u, d}}{1-e^{\left(1+2 \nu_{u, d}\right) k \pi r_{c}}}\right)^{\frac{\phi_{0}}{\pi}}
$$

where $\phi_{0}$ is the location of the CPV source $\Phi$, with the usual convention that $\phi=0(\pi)$ is 
the UV (IR) brane, and $g_{u, d}$ is the coupling strength of $\Phi$ to the bulk fermions. For $\mathcal{O}(1)$ values of $k r_{c} g_{u, d} \Phi$, this form of the Yukawas generates CKM angles that come dominantly from the down-type Yukawa and are approximately

$$
\theta_{i j} \approx\left(\frac{m_{d i}}{m_{d j}}\right)^{1-\frac{\phi_{0}}{\pi}}
$$

which works rather well for reproducing the observed values.

Another nice feature of RS flavor models in general is that the KK mode mass matrix is close to the identity. This helps with $\mathrm{CP}$ violating and flavor violating effects. Independently of $\mathrm{CP}$, the flavor model might be worth pursuing, particularly if the stringent $Z$ boson coupling constraints can be alleviated.

Finally, we compare the role of twisting in the model of [12] and our model. Twisting plays an essential role in the first model since both the up and down mass matrices can be diagonalized by different transformations at different points and it is only because of the different phase rotations required at the different points in the bulk that $\mathrm{CP}$ violation occurs. The use of twisting is a little more subtle in our case, where it generates a hierarchy in the CKM angles and furthermore sequesters $\mathrm{C}$ violation from the IR brane Yukawa couplings which is essential to suppressing strong $\mathrm{CP}$ violation.

\section{The model}

\subsection{Definition of the model}

$\mathrm{RS}$ is defined on a non-factorizable warped geometry with metric

$$
d s^{2}=e^{-2 k r|\phi|} \eta_{\mu \nu} d x^{\mu} d x^{\nu}+r^{2} d \phi^{2}
$$

where the extra dimension is an $S^{1} / Z_{2}$ orbifold of size $r$ labeled by a coordinate $\phi \in$ $[-\pi, \pi]$,such that the points $\left(x^{\mu}, \phi\right)$ and $\left(x^{\mu},-\phi\right)$ are identified. At orbifold fixed points at $\phi=0$ and $\phi=\pi$ lie the UV and IR brane, respectively. Note that this orbifold boundary condition forces either the left or right-handed zero mode of every bulk fermion wavefunction to vanish.

The singlet SM fermions, $\left(U_{L}^{\prime}, U_{R}\right)_{i}$ and $\left(D_{L}^{\prime}, D_{R}\right)_{i}$, come in Dirac multiplets that live in the bulk, while the doublets $\left(Q_{L}\right)_{i}$, are localized on the brane. Here $i$ is a flavor index and ' labels the fermion component whose zero mode is projected out by the orbifold boundary condition. Thus, zero modes exist only for $Q_{L}, U_{R}$, and $D_{R}$, and not the primed fields. The Higgs, $H$, is localized on the IR brane. Finally, we include a CP odd bulk scalar, $\Phi_{i j}$, that is localized at a position $\phi_{0}$ in the bulk and charged under flavor and acquires a complex vev that is the source of all CP phases in the theory. The action for our theory 
(neglecting kinetic terms) is

$$
\begin{aligned}
S= & S_{\text {bulk }}+S_{\text {brane }} \\
S_{\text {bulk }}= & \int d^{5} x \sqrt{G}\left\{-\bar{U}_{L}^{\prime}\left(M_{u}+g_{u} \Phi \delta\left(\phi-\phi_{0}\right)\right) U_{R}\right. \\
& \left.\quad-\bar{D}_{L}^{\prime}\left(M_{d}+g_{d} \Phi \delta\left(\phi-\phi_{0}\right)\right) D_{R}+\text { h.c. }\right\}, \\
S_{\text {brane }}= & \int d^{4} x \sqrt{-g}\left\{\bar{Q}_{L} \tilde{H} \lambda_{u} U_{R}+\bar{Q}_{L} H \lambda_{d} D_{R}+\text { h.c. }\right\},
\end{aligned}
$$

where $g_{u, d}, \lambda_{u, d}$ are numbers and $M_{u, d}$ are matrices. Note that we have assumed that CP is a valid symmetry in the UV, so all of the couplings in this action are manifestly real.

The mechanism for eliminating $\bar{\theta}$ at tree-level relies crucially on the spacetime symmetry, $\mathrm{SO}(4,2)$, which constrains the only renormalizable interaction between the CPV source $\Phi$ and a bulk fermion $\Psi$ to be of the form $\Phi_{i j}\left(\bar{\Psi}_{L i} \Psi_{R j}+\bar{\Psi}_{R i} \Psi_{L j}\right)$, so only the hermitian piece of $\Phi$ couples to the fermions in the action. If $\Phi$ is furthermore $C P$ odd, as we impose, then its couplings are further restricted:

$$
\mathcal{L} \supset g\left(\Phi_{[i j]}+\Phi_{[i j]}^{\dagger}\right)\left(\bar{\Psi}_{L i} \Psi_{R j}+\bar{\Psi}_{R i} \Psi_{L j}\right) \delta\left(\phi-\phi_{0}\right)
$$

After $\Phi$ acquires a vev, this will introduce phases and twisting in the fermion wavefunctions.

\subsection{Zero mode wavefunctions}

In this section we show that zero modes wavefunctions generically have real determinant in extra dimensional theories and establish our notation for the fermion wavefunctions that we will use in the remainder of the paper. For now, let us consider just a single massive bulk fermion flavor multiplet $\Psi_{i}$ coupled to a CP-odd bulk scalar $\Phi$; of course, our results will hold for the SM fermions, where $\Psi_{R}=U_{R}, D_{R}$ for the up-like and down-like singlets, respectively. The action is

$$
S=\int d^{5} x \sqrt{G}\left\{\frac{1}{2} i \bar{\Psi}_{i} \Gamma^{A}\left(\partial_{A}-\overleftarrow{\partial}_{A}\right) \Psi_{i}-\bar{\Psi}_{i}\left(M_{i j}+g \Phi_{i j} \delta\left(\phi-\phi_{0}\right)\right) \Psi_{j}\right\}
$$

After imposing orbifold boundary conditions on the bulk fermions, the KK reduction will contain a vector-like spectrum of KK excitations for all but a single chiral zero mode. As before, all the couplings in the above action are real because CP is preserved in the UV. We assume that $\Phi$ has some CP respecting potential, $V(\Phi)$, which is therefore even in $\Phi$, that induces spontaneous symmetry breaking, giving $\Phi$ a complex vev that sources CP violation in our theory.

We perform a standard KK reduction of the bulk fermions as follows:

$$
\Psi_{L, R}(x, \phi)_{i}=\frac{e^{2 k r|\phi|}}{\sqrt{r}} \sum_{\alpha} F_{L, R}(\phi)_{i \alpha} \times \psi_{L, R}(x)_{\alpha} .
$$

The explicit factor of $e^{2 k r|\phi|}$ is conventional, and simplifies the expressions for the normalization conditions of the wavefunctions. Let us consider the KK reduction of $\Psi$. All of the $x$ dependence lies in the (vector of) dynamical 4D fields $\psi$, while the $\phi$ dependence 
is included in the (matrix of) wavefunctions $F$. Notice that this matrix has a flavor index $i$ which runs from 1 to 3 as well as a KK mode index $\alpha$, which runs from 1 to $N$, where $N$ labels the KK mode at which the model becomes gravitationally strongly coupled. In general, it is very natural to group these KK modes in groups of threes because of the approximate flavor symmetry. For example there will always be three exactly massless zero modes (before electroweak symmetry breaking), which in the case of $\Psi=U_{R}$ correspond to the right-handed up, charm, and top. Thus, we can parse the mass matrix into a separate 3 by 3 matrix for each KK level:

$$
\begin{aligned}
F_{i \alpha} & =\{\overbrace{\left\{F_{i 1}, F_{i 2}, F_{i 3}\right\}}^{\text {zero modes }}, \overbrace{\left\{F_{i 4}, F_{i 5}, F_{i 6}\right\}}^{\text {1st excited modes }}, \ldots\} \\
& =\left\{F_{i \alpha}^{(0)}, F_{i \alpha}^{(1)}, \ldots\right\} .
\end{aligned}
$$

Using this notation, it is straightforward to refer to a particular KK mode of a particular generation: for example $F_{i 3}^{(1)}$ denotes the 3 -vector wavefunction corresponding to the first KK excitation of a third generation fermion. ${ }^{2}$ Also, note that in a theory in which all the flavors are decoupled from each other, $F^{(n)}$ is simply a diagonal matrix. In a theory in which the flavors are degenerate, $F^{(n)}$ is proportional to the identity matrix.

For each KK fermion $\left(\psi_{L, R}\right)_{\alpha}$, its wavefunction $\left(F_{L, R}\right)_{i \alpha}$ obeys the first order wave equation

$$
\left( \pm \frac{1}{r} \partial_{\phi}-\left(M+g\langle\Phi\rangle \delta\left(\phi-\phi_{0}\right)\right)\right)_{i j}\left(F_{L, R}\right)_{j \alpha}=-e^{k r|\phi|} m_{\alpha}\left(F_{R, L}\right)_{i \alpha}
$$

where $m_{\alpha}$ is the KK mass. Going to a more convenient variable [11], $t=\epsilon e^{k r|\phi|} \in[\epsilon, 1]$, where $\epsilon=e^{-k r \pi}=10^{-16}$, rescaling $F_{L, R}(\phi) \rightarrow \sqrt{k r \epsilon} F_{L, R}(t)$, and defining

$$
\begin{aligned}
\mu_{i j} & =\frac{1}{k}\left(M+g k r t\langle\Phi\rangle \delta\left(t-t_{0}\right)\right)_{i j}, \\
x_{\alpha} & =\frac{m_{\alpha}}{\epsilon k} .
\end{aligned}
$$

Now because of the orbifold boundary condition, we are forced to set either the zero mode for $\Psi_{L}$ or $\Psi_{R}$ to vanish at the orbifold fixed points $t=\epsilon, 1$. Assuming we choose to eliminate the right-handed zero mode, the left-handed zero mode then satisfies the wave equation and orthonormality condition

$$
\begin{array}{r}
\left(\partial_{t}-\frac{\mu}{t}\right) F_{L}^{(0)}=0 \\
\int_{\epsilon}^{1} d t F_{L}^{(0) \dagger} F_{L}^{(0)}=\int_{\epsilon}^{1} d t F_{R}^{(0) \dagger} F_{R}^{(0)}=\mathbf{1},
\end{array}
$$

\footnotetext{
${ }^{2}$ There is some freedom here to choose what we mean by "third generation" of the excited modes, since the mass matrix remains diagonal under permutations of any two fermion modes. One sensible choice would be to rank the "generations" by increasing mass within each KK level. Another reasonable definition would be to demand that as $\Phi$ is decreased continuously to zero, the $F$ matrices transform continuously to diagonal matrices. We choose the latter of these, though clearly none of our results depend on this choice.
} 
where we have suppressed flavor indices. Equation (3.14) can be formally integrated to obtain

$$
F_{L}^{(0)} \propto \mathcal{P} \exp \left(\int_{\epsilon}^{t} \frac{\mu\left(t^{\prime}\right)}{t^{\prime}} d t^{\prime}\right)
$$

where $\mathcal{P}$ denotes path ordering with respect to t. The proportionality constant in (3.16) is a constant hermitian matrix. We have chosen the lower bound on the integral in (3.16) to be $\epsilon$ so that the normalization matrix is close to the identity for UV localized zero modes, and thus does not have a qualitative effect on any of our discussions. We do of course include it in all of our numeric computations. Because $\mu$ is Hermitian, we see that $F_{L}^{(0)}$ is a product of infinitesimal Hermitian matrices, so it has real determinant. Thus, extra dimensions generically yield zero mode matrix wavefunctions which have real determinant.

\subsection{Approximate dependence of CKM angles on $t_{0}$}

Consider now the structure of the observed Yukawa matrices if we assume that $\Phi$ has sufficiently large entries to completely scramble the entries of any matrix that it multiplies. Let us split the path-ordered integral in equation (3.16) into three pieces:

$$
\begin{aligned}
F^{(0)}(1) & =F\left(1 ; t_{0}\right) \times \exp (\operatorname{krg} \Phi) \times F\left(t_{0} ; \epsilon\right) \\
F\left(1 ; t_{0}\right) & \equiv\left[\mathcal{P} \exp \left(\int_{t_{0}}^{1} \frac{\mu}{t^{\prime}} d t^{\prime}\right)\right]=t_{0}^{-\nu} \\
F\left(t_{0} ; \epsilon\right) & \equiv\left[\mathcal{P} \exp \left(\int_{\epsilon}^{t_{0}} \frac{\mu}{t^{\prime}} d t^{\prime}\right)\right]=\left(\frac{\epsilon}{t_{0}}\right)^{-\nu}
\end{aligned}
$$

where $\nu=M / k$ is a constant matrix. Then, $L_{u, d}$ restricted to the zero modes approximately diagonalizes

$$
Y_{u, d} Y_{u, d}^{\dagger}=F_{u, d}\left(1 ; t_{0}\right) e^{k r g \Phi} F_{u, d}\left(t_{0} ; \epsilon\right) F_{u, d}\left(t_{0} ; \epsilon\right)^{\dagger} e^{k r g \Phi} F_{u, d}\left(1 ; t_{0}\right)^{\dagger}
$$

Assume for simplicity that the nonzero entries of $\Phi$ are not so large that they contribute to the hierarchy of the fermion wavefunctions on the $\mathrm{TeV}$ brane; one could of course consider more general cases. Since $e^{k r g \Phi}$ completely mixes flavor, the above expression has the basic structure $\left(Y_{u, d} Y_{u, d}^{\dagger}\right)_{i j} \approx F_{u, d}\left(1 ; t_{0}\right)_{i k} \zeta_{k k^{\prime}} F_{u, d}\left(1 ; t_{0}\right)_{k^{\prime} j}^{\dagger}$ with $\zeta_{k k^{\prime}} \sim \mathcal{O}(1)$, which leads to mixing angles of size $\theta_{i j} \approx\left(m_{i} / m_{j}\right)^{-\left(\log t_{0}\right) /\left(k \pi r_{c}\right)}$ since $F\left(1 ; t_{0}\right)$ contains only a fraction $\log t_{0} / \log \epsilon$ of the entire fermion mass hierarchy. Because the down-type masses are less hierarchical, they will give larger contributions to the CKM angles than the uptype masses will. Then, we are left to ask whether there is a value for $t_{0}$ such that $\left(m_{d} / m_{s}\right)^{-\left(\log t_{0}\right) /\left(k \pi r_{c}\right)} \sim \lambda_{C}$ and $\left(m_{s} / m_{b}\right)^{-\left(\log t_{0}\right) /\left(k \pi r_{c}\right)} \sim \lambda_{C}^{2}$, where $\lambda_{C}=\left|V_{u s}\right|=0.23$. For instance, running the quark masses up to the scale $Q=10 \mathrm{TeV}$ allows $m_{d}=3.5 \mathrm{MeV}, m_{s}=$ $30 \mathrm{MeV}$, and $m_{b}=2.2 \mathrm{GeV}$ [22] within experimental uncertainty, and these values satisfy $\left(m_{d} / m_{s}\right)^{2} \approx\left(m_{s} / m_{b}\right)$. The further condition $\left(m_{d} / m_{s}\right)^{-\left(\log t_{0}\right) /\left(k \pi r_{c}\right)}=0.23$ implies $t_{0} \approx$ $4 \times 10^{-11}$. Such a value puts the CPV source very far in the UV and therefore will have a very small effect on the excited mode wavefunctions. Of course deviations from complete genericity are possible but as we will see unnecessary. 


\subsection{Specific values}

For concreteness, we present here a specific choice of $\Phi$ and bulk masses that roughly reproduces the Standard Model fermions masses and KM matrix.

$$
\begin{aligned}
\nu_{U} & =(-0.831,-0.665,-0.241) \\
\nu_{D} & =(-0.788,-0.734,-0.632) \\
\langle k r \Phi\rangle & =\left(\begin{array}{ccc}
0 & 1.039 i & -1.342 i \\
-1.039 i & 0 & 1.481 i \\
1.342 i & -1.481 i & 0
\end{array}\right) \\
g_{u} & =0.3 \\
g_{d} & =0.7 \\
t_{0} & =10^{-12}
\end{aligned}
$$

These numbers are not intended to be an indication of what parameters are most expected given some prior distribution. Rather, we want to indicate what is possible with $\mathcal{O}(1) \mathrm{UV}$ parameters. We set the KK scale $\mu_{\mathrm{TeV}} \equiv \epsilon k$ to be $15 v$, where $v$ is the Higgs vev $246 \mathrm{GeV}$, so that the KK gauge bosons are at $\sim 9 \mathrm{TeV}$, in order to demonstrate that $\bar{\theta}$ is sufficiently small in this case. As we discuss in section Q, electroweak constraints force $\mu_{\mathrm{TeV}}$ to be around $20 v$, so that $\bar{\theta}$ does not provide the strongest constraint on the model.

We obtain the following quark masses for the zero mode and first excited modes:

$$
\begin{aligned}
& m_{u}=(1.6 \mathrm{MeV}, 420 \mathrm{MeV}, 176 \mathrm{GeV}, 9.8 \mathrm{TeV}, 10.3 \mathrm{TeV}, 10.7 \mathrm{TeV}) \\
& m_{d}=(3.5 \mathrm{MeV}, 41 \mathrm{MeV}, 2.1 \mathrm{GeV}, 9.6 \mathrm{TeV}, 10.1 \mathrm{TeV}, 10.4 \mathrm{TeV})
\end{aligned}
$$

The absolute value of the KM matrix entries are

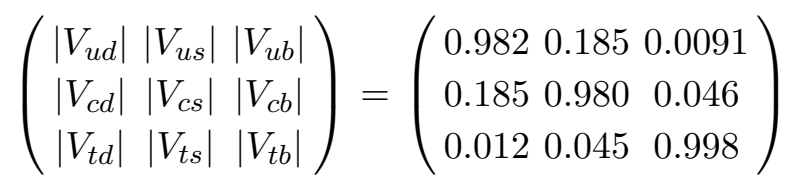

and the rephasing invariant $\Delta^{(4)} \equiv \operatorname{Im}\left(V_{11} V_{12}^{\dagger} V_{22} V_{21}^{\dagger}\right)=7.6 \times 10^{-5}$ Because of mixing with the excited modes, the CKM matrix for the zero modes is not exactly unitary. The first and second rows satisfy

$$
\begin{array}{r}
\left|V_{u d}\right|^{2}+\left|V_{u s}\right|^{2}+\left|V_{u b}\right|^{2}-1=-0.0021 \\
\left|V_{c d}\right|^{2}+\left|V_{c s}\right|^{2}+\left|V_{c b}\right|^{2}-1=-0.0024
\end{array}
$$

which is at the limit of current bounds on unitarity $[2]^{3}$ and decreases proportional to $1 / m_{\mathrm{KK}}^{2}$. This is further enhanced by $\sum_{n=1}^{\infty} n^{-2}=\pi^{2} / 6$ when we include several higher KK modes.

\footnotetext{
${ }^{3}$ See their section 11.4
} 


\subsection{Fermion wavefunction dependence on the bulk masses}

The dependence of the KK fermion wavefunctions on their bulk masses will be important for all of our phenomenological constraints. In particular, as we will now discuss, the value of the $\mathrm{KK}$ fermion wavefunctions in the IR is very insensitive to flavor violation from sources in the bulk, and especially in the UV.

Most of the qualitative features of the twisted excited mode wavefunctions can be understood in the absence of twisting. The method we use for solving the wavefunctions with and without twisting is reviewed in appendix A. To begin, we note that in the single-generation case, the left-handed mode wavefunctions are given approximately by

$$
f_{L}(t)=\left\{\begin{array}{l}
\frac{\sqrt{2 t} J_{-\frac{1}{2}+\nu}(x t)}{J_{-\frac{1}{2}+\nu}(x)}, \nu>-\frac{1}{2} \\
-\frac{\sqrt{2 t} J_{\frac{1}{2}-\nu}(x t)}{J_{\frac{1}{2}-\nu}(x)}, \nu<-\frac{1}{2}
\end{array}\right.
$$

except for the zero mode, for which $f_{R}$ vanishes and it is easy to solve for $f_{L}$ :

$$
f_{L}^{0}=\sqrt{\frac{1+2 \nu}{1-\epsilon^{1+2 \nu}}} t^{\nu}
$$

An important point is that the wavefunctions of the excited modes depend weakly on the values of the $\nu$ 's, which are the source of flavor-breaking. Indeed, up to $\mathcal{O}(\epsilon)$ corrections, the KK modes at the $\mathrm{TeV}$ brane are all $\sqrt{2}$, independent of the value of $\nu$. This plays an important role in suppressing flavor-violation, since it reduces the flavor-violation in the KK mode wavefunctions.

The reason for this is that the behaviour near the UV boundary approximately fixes the phase of the $f_{L}$ modes, so that there is no $\nu$-dependent phase shift, as there would be if we had constant bulk masses $\nu$ in flat space. This follows from the fact the $f_{L}$ are a linear combination of a regular mode and a singular mode in the $t \sim \epsilon$ region, and the regular mode vanishes at $t \rightarrow 0$. The singular mode is therefore very suppressed, and the turnaround point (where $f_{L}^{\prime}(t)=0$ ) from singular to regular mode occurs at very small $t$ :

$$
t_{\text {turnaround }}=\left(\frac{4 \nu^{2}-1}{x^{2}} \epsilon^{-2 \nu-1}\right)^{\frac{1}{1-2 \nu}} \quad\left(\nu<-\frac{1}{2}\right)
$$

so in the warped space case, the phase shift due to $\nu$ is small and suppressed by powers of $\epsilon$. Another difference is that there is less variation required among the $\nu$ 's in warped space, because of an enhancement from $k r_{c} \sim 10$. The zero modes at the IR brane in warped space are

$$
\begin{aligned}
f^{(0)}(t=1) & =\sqrt{\frac{2 \nu+1}{1-e^{-(2 \nu+1) k r_{c} \pi}}} \\
& \approx \sqrt{|2 \nu+1|} e^{-\left|\nu+\frac{1}{2}\right| k r_{c} \pi} \quad\left(\nu<-\frac{1}{2}\right)
\end{aligned}
$$

and therefore we need $\nu+\frac{1}{2}$ 's of size $\frac{1}{k r_{c} \pi} \log \left(\left(m_{t} / m_{u}\right)^{1 / 2}\right)=0.17$. This might perhaps be considered a little more tuned than in flat space, since the $\nu$ 's need to fall close to $-\frac{1}{2}$ in 
warped space. However, once the $\nu$ 's are fixed from experimental constraints, the KK mode wavefunctions are more flavor universal which leads to much weaker phenomenological constraints.

\section{Interactions in the KK reduced theory}

\subsection{Yukawa interactions}

In this subsection we determine the flavor structure of the Yukawa interactions. In particular, we will show that just like the charged $W$ interactions, the Yukawas can be written entirely in terms of the CKM matrix.

To begin, we plug back in to eq. (3.2), and find that the effective 4D Yukawa interactions between zero modes become ${ }^{4}$

$$
S_{4 \mathrm{D} \text { Yukawa }}=\int d x^{4} \sqrt{-g}\left\{\bar{q}_{L} \tilde{h} Y_{u}^{(0)} u_{R}^{(0)}+\bar{q}_{L} h Y_{d}^{(0)} d_{R}^{(0)}\right\}
$$

where $Y_{u}^{(0)}$ and $Y_{d}^{(0)}$ are defined by

$$
\begin{aligned}
& Y_{u}^{(0)}=\left.\lambda_{u} F_{u}^{(0)}\right|_{\phi=\pi}, \\
& Y_{d}^{(0)}=\left.\lambda_{d} F_{d}^{(0)}\right|_{\phi=\pi} .
\end{aligned}
$$

and $\lambda_{u, d}$ are just real numbers. As we showed explicitly in the previous section, in extra dimensional scenarios, zero mode wavefunctions $F_{u, d}^{(0)}$ are complex but have real determinant. Thus, from eq. (1.2) we see that $\bar{\theta}$ vanishes at tree-level while $\mathrm{CP}$ violation in the weak interactions does not. In order to calculate the radiative corrections to this treelevel result, we need to work out the interactions of the effective theory. The KK tower of fermions has interactions that are simple extensions of those of the Standard Model, though with some non-trivial consequences. In the SM, the only source of masses is the Yukawa interactions, and so going to the mass eigenbasis pushes all $\mathrm{CP}$ violation into a single unitary KM matrix in the gauge kinetic terms. In contrast, extra dimensional models contain Yukawa interactions that mix different KK levels, as well as KK mass eigenvalues from the dimensional reduction.

Let us consider the effective $4 \mathrm{D}$ mass matrix, limiting the following discussion to the zero modes and first excited modes. Our arguments can be extended easily to the entire KK tower. We will group the left and right-handed up-like modes together

$$
\begin{aligned}
& u_{L}=\left(u_{L}^{(0)}, u_{L}^{\prime(1)}\right), \\
& u_{R}=\left(u_{R}^{(0)}, u_{R}^{(1)}\right),
\end{aligned}
$$

where each is a vector of six fields: three for the zero mode, and three for first excited modes. The "zero" mode for $u_{L}$ is just the brane-localized up-type component of the

\footnotetext{
${ }^{4}$ Here we denote fields in the KK reduced description by lower-case letters, as in equation (3.8). For example, $u_{R}^{(i)}$ is in the KK tower of the 5D field $U_{R}$, while $H$ and $h$ are the same since $H$ is a brane-localized field in the $5 \mathrm{D}$ description.
} 
doublet $q_{L}$, whereas $u_{R}^{(0)}, u_{L}^{\prime(1)}, u_{R}^{(1)}$ are zero and KK modes of the bulk field $U_{R}$ (recall that there are no bulk $q_{L}$ 's). Note that the ordering has been chosen so that the mass matrix is approximately diagonal in this basis. The effective 4D mass matrix receives contributions from Yukawa interactions and KK masses:

$$
\begin{aligned}
S_{4 \mathrm{D} \text { mass }} & \supset \int d^{4} x\left\{\bar{u}_{L} M_{u} u_{R}\right\}, \\
M_{u} & =v Y_{u}+m_{u}, \\
Y_{u} & =\left(\begin{array}{cc}
F_{u}^{(0)} & F_{u}^{(1)} \\
0 & 0
\end{array}\right), \\
m_{u} & =\left(\begin{array}{cc}
0 & 0 \\
0 & m_{u}^{(1)}
\end{array}\right)
\end{aligned}
$$

where $m_{u}$ is a diagonal matrix containing the excited mode masses that arise from the KK reduction in the absence of the Higgs interactions. We will refer to this basis as the "KK basis". Moreover, unlike in the SM, since the $Y_{u}$ does not commute with $M_{u}$, the Yukawa matrix for the KK tower is not diagonal in the mass eigenbasis.

Defining $Y_{d}, M_{d}, m_{D}$ analogously, we perform bi-unitary transformations that diagonalize the mass matrices:

$$
\begin{aligned}
L_{u} M_{u} R_{u}^{\dagger} & =\mu_{u}, \\
L_{d} M_{d} R_{d}^{\dagger} & =\mu_{d},
\end{aligned}
$$

where $\mu_{u, d}$ are the (real and diagonal) physically observable fermion mass matrices. The Yukawas in the mass eigenbasis can be slightly simplified as follows. It will be useful here and throughout to define the projection matrix onto zero modes:

$$
P_{0} \equiv\left(\begin{array}{ll}
1 & 0 \\
0 & 0
\end{array}\right)
$$

It is clear that $v Y_{u, d}=P_{0} M_{u, d}$. The transformation of this equation into the mass eigenbasis takes the form

$$
\begin{aligned}
& v L_{u} Y_{u} R_{u}^{\dagger}=L_{u}\left(\begin{array}{ll}
1 & 0 \\
0 & 0
\end{array}\right) L_{u}^{\dagger} \mu_{u} \\
& v L_{d} Y_{d} R_{d}^{\dagger}=L_{d}\left(\begin{array}{ll}
1 & 0 \\
0 & 0
\end{array}\right) L_{d}^{\dagger} \mu_{d}
\end{aligned}
$$

Notice the presence of only $L$ 's on the r.h.s. . This has the important consequence, mentioned in the introduction, that flavor-changing in the $W$ and Higgs interactions is controlled by a single CKM matrix

$$
\begin{aligned}
V_{\mathrm{CKM}} & =L_{u} P_{0} L_{d}^{\dagger} \\
V_{u u} & =L_{u} P_{0} L_{u}^{\dagger}=V_{\mathrm{CKM}} V_{\mathrm{CKM}}^{\dagger} \\
V_{d d} & =L_{d} P_{0} L_{d}^{\dagger}=V_{\mathrm{CKM}}^{\dagger} V_{\mathrm{CKM}}
\end{aligned}
$$


Equation (4.13) can therefore be rewritten as

$$
v Y_{u, d}=V_{u u, d d} \mu_{u, d}
$$

for interactions with neutral Higgses. We will find it convenient to work in a gauge where the longitudinal modes of $W^{ \pm}$are kept explicitly as the goldstone modes $h^{ \pm}$. In the mass eigenbasis, the $h^{ \pm}$interactions satisfy $v Y_{d}=V_{\mathrm{CKM}} \mu_{d}, v Y_{u}=V_{\mathrm{CKM}}^{\dagger} \mu_{u}$.

This is very similar to the Standard Model, where all flavor-changing is controlled by a single CKM matrix. In particular, this is a sufficient condition for radiative corrections to $\bar{\theta}$ and dimension-5 EDM operators to vanish at 1-loop, as we will discuss in section 5.2. We note that, unlike in the Standard Model, $V_{\text {CKM }}$ is not unitary. However, in the limit that the KK masses are large, mixing between the zero modes and KK modes approaches zero. In this limit, $V_{\mathrm{CKM}}$ restricted to the $\mathrm{KK}$ modes vanishes, and restricted to the zero modes is exactly the unitary CKM matrix of the Standard Model.

The structure of the mass matrices has an interesting and important consequence for the mixing between zero modes and KK modes. Compared to models with doublets localized in the UV, the mixing between the doublet zero modes and KK modes is greatly enhanced, while that between singlet zero modes and KK modes is greatly suppressed. The reason is that $L_{u}$ diagonalizes $M_{u} M_{u}^{\dagger}$ while $R_{u}$ diagonalizes $M_{u}^{\dagger} M_{u}$, and these have different orders of magnitude in the off-diagonal components between zero modes and KK modes. More precisely, $M_{u} M_{u}^{\dagger}$ has zero-mode-KK-mode mixing from the block matrix $v F_{u}^{(1)} M_{u}^{(1)}$ while $M_{u}^{\dagger} M_{u}$ has zero-mode-KK-mode mixing from the block matrix $\left(v^{2} F_{u}^{(0) \dagger} F_{u}^{(1)}\right)$. As we review in section A.1, the size of excited mode wavefunctions in $\mathrm{RS}$ in is $F_{u}^{(1)} \sim \sqrt{2}$. Thus, the mixing for the doublets is therefore of the order $\left(L_{u}\right)_{i j} \sim \sqrt{2} v / m_{\mathrm{KKf}}$, while that for the singlets is of the order $\left(R_{u}\right)_{i j} \sim \sqrt{2}\left(v m_{0} / m_{\mathrm{KKf}}^{2}\right)$, where $m_{0}$ is the zero-mode mass. ${ }^{5}$

\section{CP and flavor constraints}

\subsection{Contributions to $\Phi$ interactions from higher-dimensional operators}

The vanishing of $\bar{\theta}$ at tree-level depended crucially on the form of the fermion couplings to $\Phi$. For this reason, one might worry that higher-dimensional operators could destroy this result. This is not the case, however. The only higher-dimensional interaction $\Phi$ can have with a fermion bilinear other than $\bar{\Psi} \Psi$ is through

$$
\mathcal{L} \supset F(\Phi)_{i j} D_{M} \bar{\Psi}_{i} \Gamma^{M} \Psi_{j}
$$

This does not contribute to the $\Psi$ wavefunction for the following reason. Since $\Phi$ vanishes on the boundary, all boundary terms involving $\Phi$ vanish as well. Under the assumption that $\langle\Phi\rangle$ and the bulk masses (the only sources of flavor symmetry breaking in our model) are generated spontaneously, then $D_{M} \bar{\Psi}_{i} \Gamma^{M} \Psi_{j}$ is the divergence of a current that is conserved up to a chiral anomaly on the boundary [37]. Since the $\Phi$ field vanishes on the boundary,

\footnotetext{
${ }^{5}$ We have introduced the notation $m_{\mathrm{KKf}}$ to indicate the mass of KK fermions and $m_{\mathrm{KKg}}$ for the gauge bosons.
} 
and $D_{M} J_{i j}^{M}$ vanishes off the boundary, these higher dimensional operators do not contribute to the fermion wavefunctions. ${ }^{6}$

In addition, one might worry that $\Phi$ could couple directly to $G \tilde{G}$ through higher dimensional operators of the form

$$
\mathcal{L} \supset G_{A B} G_{C D} D_{E} F(\Phi) \epsilon^{A B C D E},
$$

where $F(\Phi)$ is a function of $\Phi$ and the form of this operator is again constrained by the $5 \mathrm{D}$ AdS isometries. However, restricting to the gluon zero modes, which have flat wavefunctions, this operator is a total derivative of $\Phi$ and therefore integrates to a vanishing boundary term in the low-energy theory.

Finally, as we have already mentioned in the introduction, sequestering $\Phi$ from the $\mathrm{TeV}$ brane eliminates the higher-dimensional operator $\Phi_{i j} \bar{Q}_{L i} H U_{R j}$.

\subsection{The EDM operator and $\bar{\theta}$ at one loop}

As we saw in section 1 , extra dimensional models generate Hermitian bulk fermion wavefunctions which force $\bar{\theta}$ to vanish at tree level. In this section, we show that by also taking the quark doublets to be brane-localized, we can eliminate $\bar{\theta}$ (and any EDMs) at one loop level.

To begin, let us consider the higher dimension operator

$$
\mathcal{O}_{\mathrm{EDM}}=\frac{\bar{d}_{L} \gamma^{\mu \nu} \tilde{F}_{\mu \nu} d_{R}}{\Lambda_{\mathrm{EDM}}}
$$

which is phenomenologically relevant for the neutron EDM, and is constrained by experiment to be $d_{n}<10^{-24} e \mathrm{~cm} \mathrm{[24].}$

In the past this EDM operator has been quite constraining for models embedded in warped backgrounds. For example, in [25] the authors assume anarchic brane Yukawas along with bulk doublet and singlet fermions, and as a result the EDM operator is generated at one loop via a virtual Higgs. Consequently, the KK gauge boson masses are constrained to be $\gtrsim 10 \mathrm{TeV}$ for that model. As we will now show, with the quark doublets on the IR brane, this one loop contribution vanishes.

Even without evaluating the one loop diagram, we can immediately see that this contribution explicitly vanishes because its corresponding spurion vanishes. The spurion for the down-type quark EDMs is fixed by flavor symmetries to be

$$
d_{i} \propto \operatorname{Im}\left(L_{d}\left(Y_{u}\left(M_{u}^{\dagger} M_{u}\right)^{n} M_{u}^{\dagger}+Y_{d}\left(M_{d}^{\dagger} M_{d}\right)^{n} M_{d}^{\dagger}\right) Y_{d} R_{d}^{\dagger}\right)_{i i}, \quad n=0,1, \ldots
$$

where the $i i$ subscript labels the down-type quarks (zero and excited modes). Also, here $n$ denotes some number of mass-squared insertions.

\footnotetext{
${ }^{6}$ Strictly speaking, the brane Yukawas explicitly break the $\mathrm{U}(3)^{3}$ flavor symmetry corresponding to this current down to the diagonal subgroup. However, this breaking occurs on the $\mathrm{TeV}$ brane, sequestered from the $\Phi$ field, and is therefore not a problem.
} 
This contribution vanishes for essentially the same reason that it vanishes in the Standard Model. Using equations (4.10)-(4.17), we see that the above term is

$$
d_{i} \propto \operatorname{Im}\left(V_{\mathrm{CKM}}^{\dagger} \mu_{u}^{2 n+2} V_{\mathrm{CKM}} \mu_{d}+V_{d d} \mu_{d}^{2 n+2} V_{d d} \mu_{d}\right)_{i i} \quad n=0,1, \ldots
$$

It follows from the fact that $V_{i j} V_{j i}^{\dagger}=\left|V_{i j}\right|^{2}$ is real for any matrix $V$ (no sum on $i, j$ implied) that the above term is also real.

Actually, the vanishing of EDMs at one loop also implies the vanishing of $\bar{\theta}$ at one loop. This is important because in most models of spontaneous CP breaking, even if $\bar{\theta}$ vanishes at tree level, one-loop radiative corrections force the scale of $\mathrm{CP}$ breaking up to near the GUT scale. To see that IR brane-localized fermions remedy this, recall that 29]

$$
\begin{aligned}
\bar{\theta} & =\arg \operatorname{det}\left(M_{u}+\delta M_{u}\right) \operatorname{det}\left(M_{d}+\delta M_{d}\right) \\
& \approx \operatorname{Im} \operatorname{Tr}\left(\delta M_{u} / M_{u}+\delta M_{d} / M_{d}\right)+\ldots
\end{aligned}
$$

where the $\delta$ 's denote contributions from one loop corrections, and we have assumed that $\arg \operatorname{det}\left(M_{u} M_{d}\right)$ vanishes because of hermitian bulk fermion wavefunctions. the EDM operator, the contribution to $\bar{\theta}$ from, say the down-type quarks, is proportional to a spurion:

$$
\begin{aligned}
\bar{\theta} & =\operatorname{Im} \operatorname{Tr}\left(\left(Y_{u}\left(M_{u}^{\dagger} M_{u}\right)^{n} M_{u}^{\dagger}+Y_{d}\left(M_{d}^{\dagger} M_{d}\right)^{n} M_{d}^{\dagger}\right) Y_{d} M_{d}^{-1}\right), \quad n=0,1, \ldots \\
& =\sum_{i} \frac{d_{i}}{\left(\mu_{d}\right)_{i}}=0 .
\end{aligned}
$$

Contributions to the EDMs and $\bar{\theta}$ from one loop diagrams with $\mathrm{KK} \mathrm{U}(1)_{Y}$ bosons vanish via similar arguments. Moreover, loops of $\mathrm{SU}(2)$ weak gauge bosons do not contribute because they do not couple to right-handed fields. Again, this is only the case because the left-handed doublets are IR-localized. If this were not the case, then weak bosons would couple to their right-handed bulk partners and contribute at one loop.

\section{$5.3 \bar{\theta}$ at two loops from $W$ 's and higgses}

Thus, we have shown that the leading contributions to the EDMs and $\bar{\theta}$ enter at two loops. At this loop level, an evaluation of $\bar{\theta}$ becomes far more complicated because of the proliferation of diagrams, and so we find it useful to first analyze the contributions in terms of their spurions.

We will address diagrams with KK gluon and hypercharge boson $(B)$ contributions in the next subsection. Such contributions are sufficiently small only in the small $t_{0}$ limit where the KK fermion wavefunctions are diagonal. We first analyze the contributions from diagrams with $W^{ \pm}, W^{3}$, and Higgses, and zero mode gluons and $B$ 's. In this case, the vertices are all controlled by a single generalized CKM matrix $V_{\mathrm{CKM}}$, and their contributions to $\bar{\theta}$ are sufficiently small even for $t_{0} \sim 1 / 100$.

To begin, we note that any diagram renormalizing $M_{u, d}$ necessarily ends with a righthanded fermion line following an interaction with a Higgs or a neutral gauge boson (e.g. gluon or $\mathrm{U}(1)_{Y} \mathrm{KK}$ mode, $\left.B^{(1)}\right)$. However, the zero mode gluon and $B$ interactions are flavor-respecting so we can ignore their contributions. Also, we note that the right-most 
interaction cannot be with the $W^{ \pm}, W^{3}$ or their KK excitations because these fields do not couple to any right-handed fields.

Thus the dominant loop contributions to $\bar{\theta}$ will be from virtual $W$ and $h$ exchange, and the final vertex insertion should be a Yukawa. We have already seen in section 4.1 that all $\mathrm{W}$ and Yukawa contributions are formed out of quark masses and the (generalized) CKM matrices:

$$
\begin{aligned}
V_{\mathrm{CKM}} & =L_{u} P_{0} L_{d}^{\dagger} \\
V_{u u} & =V_{\mathrm{CKM}} V_{\mathrm{CKM}}^{\dagger} \\
V_{d d} & =V_{\mathrm{CKM}}^{\dagger} V_{\mathrm{CKM}} .
\end{aligned}
$$

For example, in mass eigenstate, the Yukawa interaction between two up-type quarks and a Higgs field can be written as $L_{u} Y_{u} R_{u}^{\dagger}=L_{u} P_{0} L_{u}^{\dagger} L_{u} M_{u} R_{u}^{\dagger} / v=V_{u u} \mu_{u} / v$. Note that written in terms of $V$ and $\mu$, each Higgs propagator is necessarily accompanied by two factors of $1 / v$.

It is important to notice that the right-handed transformation matrices $R_{u, d}$ do not explicitly appear anywhere, since in mass eigenstate basis they are all absorbed into mass insertions. This implies that the contributions to $\bar{\theta}$ can be written completely in terms of the fermion masses, the Higgs vev $v$, the generalized CKM's $V_{u u}, V_{d d}, V_{\mathrm{CKM}}$, and propagators. No other terms appear. We emphasize the similarity of the above simplification to that of the Standard Model, where all CP and flavor violation appears through the CKM matrix and quark masses. Nonetheless, there are two essential differences that complicate the analysis: 1) there are heavy KK fermions above the $W$ and $h$ masses so additional factors of their masses do not suppress the overall contribution, and 2) the CKM matrix is not unitary because the doublet zero modes mix with left-handed KK singlets. Thus, $\bar{\theta}$ renormalization occurs at two-loops instead of three-loops as in the SM.

There are four distinct topologies of two-loop diagrams with $W$ and/or Higgs loops that contribute to $\bar{\theta}$, as shown in figure 2: two Higgs lines, either nested or crossed, and a Higgs line and a $\mathrm{W}$ line, either nested or crossed. The actual size of the largest contributions depends on how close the excited mode wavefunctions are to being proportional to the identity matrix, and therefore on the position $t_{0}$ of the CPV field.

Contributions to $\bar{\theta}$ are IR convergent, and dominated by momenta $\sim m_{\mathrm{KKf}}$. It is straightforward though somewhat laborious to calculate numerically the size of contributions to $\bar{\theta}$ from $W, h$ loops. The largest contribution comes from the crossed higgs diagram with a charged Higgs and a neutral Higgs, and a helicity flip on the first fermion line. This contribution is given schematically by the two loop integral: ${ }^{7}$

$$
\delta \bar{\theta}=v^{-4} \operatorname{Tr} \int\left(d^{4} p\right)^{2} V_{f_{1} f_{2}} \frac{m_{f_{2}}^{2}}{p^{2}-m_{f_{2}}^{2}} V_{f_{2} f_{3}} \frac{\left(p^{\mu} \gamma_{\mu}-m_{f_{3}}\right) m_{f_{3}}^{2}}{p^{2}-m_{f_{3}}^{2}} V_{f_{3} f_{1}} \frac{p^{\mu} \gamma_{\mu}-m_{f_{1}}}{p^{2}-m_{f_{1}}^{2}}\left(\frac{1}{p^{2}-m_{h}^{2}}\right)^{2},
$$

\footnotetext{
${ }^{7}$ The following expression has only three interactions because we have canceled away the factor of $Y_{u, d} M_{u, d}^{-1}$ in the $\bar{\theta}$ expression
} 

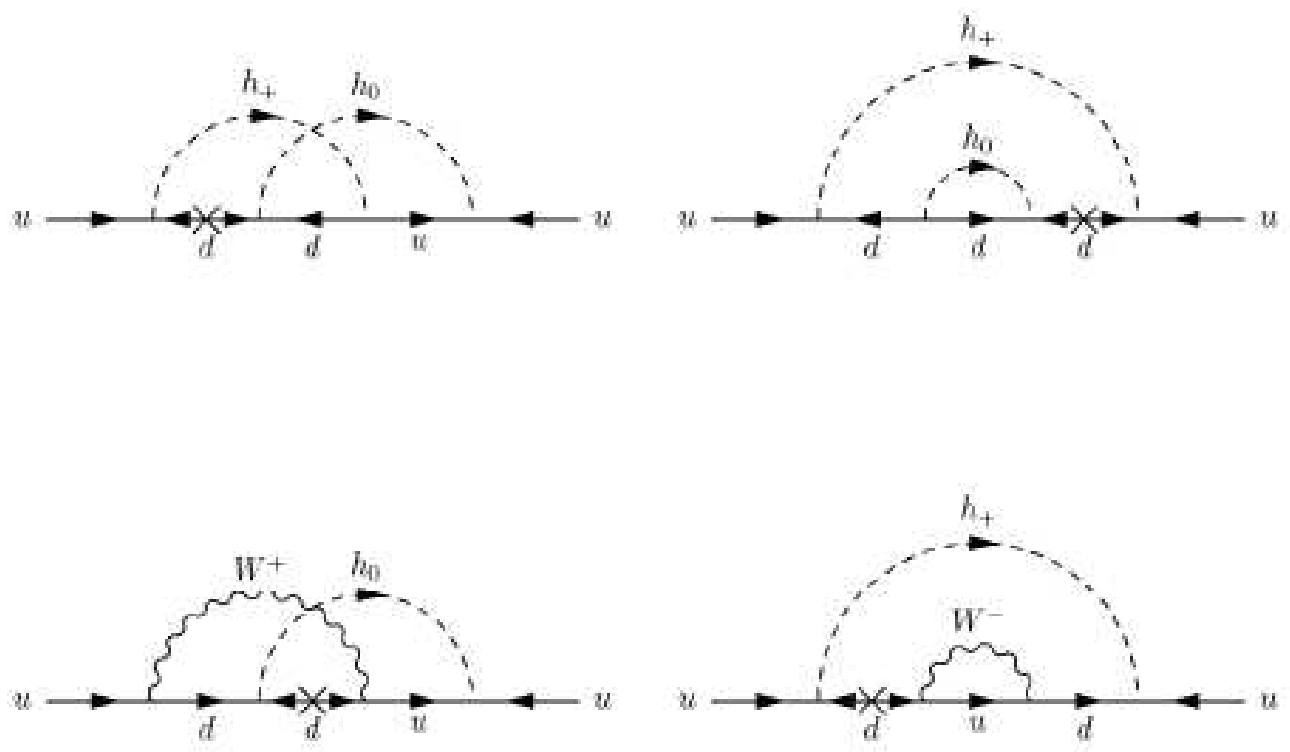

Figure 2: There are four topologies of two-loop diagrams that give the dominant contribution to $\bar{\theta}$, shown above. The largest of these corrections come from the crossed-neutral-higgs-charged-higgs (upper left).

where the $f_{i}$ run over the zero and excited mode fermions. Since $f_{i}$ run over up-like and down-like quarks, $V$ denotes the appropriate generalized CKM matrix for its given indices. For example, $V_{f_{1} f_{2}}$ where $f_{1}$ and $f_{2}$ are both up-like denotes the $f_{1} f_{2}$ component of $V_{u u}$, etc. Here we have chosen to completely ignore the momentum structure of the fermion propagators, choosing instead to emphasize the parametric dependence of the integral. Also, note the two factors $m_{f_{2}}$ in the first propagator, one of which arises from the mass insertion, and the other which arises from the rewriting a Yukawa vertex as a CKM multiplied by a mass. Both factors of $m_{f_{3}}$ come from this rewriting of neighboring Yukawa interactions. As in any diagram with two Higgs loops, there is a factor of $1 / v^{4}$. All diagrammatic contributions will go parametrically as products of masses and generalized CKMs.

The largest contribution from equation (5.12) comes from when $f_{1,2}$ are down-like and $f_{3}$ is up-like (otherwise one requires three different generations to get a phase and therefore more mixing angles). Thus, the three $V$ 's in the diagram are $V_{u d}, V_{d d}$, and $V_{d u}$. If any of the masses in the numerator come from zero modes, then this contribution is immediately very suppressed. As a result, since the contribution is maximal, then $f_{2,3}$ must be KK modes. Consider first the possibility that the remaining fermion line is also a KK mode. Then, the entire diagram is suppressed by (at least)

$$
\delta \bar{\theta} \lesssim\left(m_{\mathrm{KKf}} / v\right)^{4} \operatorname{Im} \sum_{f_{1,2,3} \in \mathrm{KKf}} \frac{1}{(4 \pi)^{4}}\left(V_{u d}\right)_{f_{1} f_{2}}\left(V_{d d}\right)_{f_{2} f_{3}}\left(V_{d u}\right)_{f_{3} f_{1}}
$$

where the fermions are summed only over KK modes. As it turns out, this on its own is 
enough to be below experimental bounds. When $f_{i}, f_{j}$ are KK modes, $V_{f_{i}, f_{j}} \sim\left(v / m_{K K f}\right)^{2}$, as follows from the fact that $V=L P_{0} L^{\dagger}$ and $L$ contains zero-mode-KK-mode mixing of order $v / m_{\mathrm{KKf}}$. This suppression and the loop factor just by themselves already result in a suppression of $(1 / 4 \pi)^{4}\left(m_{\mathrm{KKf}} / v\right)^{4}\left(v^{2} / m_{\mathrm{KKf}}^{2}\right)^{3} \sim 3 \times 10^{-7}$. There is additional suppression from the fact that $f_{1,2,3}$ must all be different, or else the product of $V$ 's has no imaginary piece. This results in a suppression from mixing between generations. We compute this suppression numerically. It is due to the fact that the KK mode wavefunctions $F_{u, d}^{(1)}$ become flavor-symmetric as $t_{0} \rightarrow 0$. We find $V_{f_{1} f_{2}} / V_{f_{1} f_{1}}$ decreases to zero approximately as $t_{0}^{1 / 2}$ (or faster in some cases) and thus one requires only $t_{0} \lesssim 10^{-4}$ in order to put the above corrections to $\bar{\theta}$ safely below experimental constraints. Finally, there is an additional suppression beyond what is contained in equation (5.13), due to a partial cancellation from the interchange of two KK modes. That is, $\left(V_{u d}\right)_{f_{1} f_{2}}\left(V_{d d}\right)_{f_{2} f_{3}}\left(V_{d u}\right)_{f_{3} f_{1}}+f_{1} \leftrightarrow f_{2}$ is real. If the KK mode masses were exactly equal, this cancellation would be exact; as it is, it implies only a cancellation of about $\left|\left(m_{f 1}-m_{f 2}\right) / m_{f 1}\right| \sim 1 / 10$.

Similar but slightly different considerations imply that the contribution with a single zero mode internal quark is roughly the same size. That is, say $f_{1}=(u, c, t)$. As before, the dominant contribution must have two different KK modes or the phases cancel. There is also a quite drastic suppression from the fact that the zero mode masses are much smaller than the typical momenta, so the zero mode propagators are approximately flavor respecting. Summing over them therefore approximately removes phases. In particular, this means that the contribution from a single zero mode internal quark is approximately equal to the contribution from all $\mathrm{KK}$ mode quarks, since the sum $\left.\sum_{j}\left(V_{d u}\right)_{K K_{1}, j}\left(V_{u d}\right)_{j, K K_{2}}\left(V_{d d}\right)_{K K_{2}, K K_{1}}\right)=\left(V_{d u} V_{u d}\right)_{K K_{1}, K K_{2}}\left(V_{d d}\right)_{K K_{2}, K K_{1}}$ is real. Putting in the weak $m$-dependence in the fermion propagators then implies that these two contributions are roughly equal in magnitude rather than exactly equal in magnitude (and opposite in sign). So the contributions with zero mode internal quarks are small as well. We therefore find that the size of radiative corrections to $\bar{\theta}$ from $W$ and higgs loops is

$$
\delta \bar{\theta} \sim 3 \times 10^{-8} t_{0}
$$

We note that this result applies when the $\mathrm{SU}(2)_{L}$ gauge group is confined to the IR brane. When $\mathrm{SU}(2)_{L}$ is in the bulk, there are $W$ KK gauge bosons that couple more strongly to the $\mathrm{TeV}$ brane by a factor of $\sqrt{2 k r_{c} \pi}$ than the zero mode KK gauge bosons do. Hence the coupling is enhanced by a factor of $g_{w} \sqrt{2 k r_{c} \pi}$ over the Yukawa interactions, and the radiative corrections are approximately

$$
\delta \bar{\theta} \sim 2 \times 10^{-6} t_{0}
$$

which is below the bound for $t_{0} \lesssim 10^{-4}$. In the next section, we will consider bigger contributions to $\bar{\theta}$. The arguments in this section relied upon the fact that all CPV was controlled in the Higgs and $W$ interactions by a single $V_{\mathrm{CKM}}$ matrix. However, the KK gluons introduce new sources of CPV that are not controlled by a single $V_{\text {CKM }}$ matrix. In the next section, we will provide a different analysis of the two-loop corrections to $\bar{\theta}$ that will be completely general and applies to all two-loop radiative corrections, gluons as well as $W$ and Higgs, but which is valid only in the small $t_{0}$ limit. 


\section{$5.4 \bar{\theta}$ from flavor spurions}

In this subsection we present the estimate of the contributions to $\bar{\theta}$ in the small $t_{0}$ limit from two loop diagrams containing neutral currents. The estimate is based on a flavor spurion analysis we describe in detail in appendix B. Our method is also applicable to diagrams containing charged currents.

In the small $t_{0}$ limit, the full mass matrices take the form

$$
M_{u, d}=\left(\begin{array}{cc}
F_{u, d}^{(0)} & \sqrt{2} \mathbf{1} \\
\mathbf{0} & M_{u, d}^{(1)}
\end{array}\right)
$$

All of the flavor-mixing effects manifestly arise from the $F_{u, d}^{(0)}$ matrices. For convenience, we define $f_{u, d}^{(0)}=L_{u, d}^{(0)} F_{u, d}^{(0)} R_{u, d}^{(0) \dagger}$ as the matrix of eigenvalues of $F_{u, d}^{(0)}$, proportional to the zero mode masses. It is straightforward to compute numerically that the left-handed diagonalization matrices $L_{u, d}$ for the full mass matrix then take the form

$$
L_{u, d}=\left(\begin{array}{cc}
L_{u, d}^{(0)} & -L_{u, d}^{(0)} \frac{v}{m_{\mathrm{KKf}}} \\
\frac{v}{m_{\mathrm{KKf}}} & \mathbf{1}
\end{array}\right)
$$

plus small deviations. The lower left-hand block of the above matrix is only diagonal and not proportional to the identity matrix because there are small $(\mathcal{O}(10 \%))$ splittings in the KK fermion masses. We find numerically that given the above structure for $M_{u, d}$ and the Standard Model values for the zero mode masses, deviations from equation (5.17) are at most $\mathcal{O}\left(10^{-13}\right)$ in $L_{u}$ and even smaller in $L_{d}$. Naively, the contributions to $\bar{\theta}$ from $L_{u, d}$ of the form (5.17) are much greater than $10^{-10}$. However, as we show in appendix B, our final result for the size of $\bar{\theta}$ in the small $t_{0}$ limit assuming $L_{u, d}$ takes exactly the form 5.17) will be very small $\left(\mathcal{O}\left(10^{-15}\right)\right)$. In fact it is exactly the $O\left(10^{-13}\right)$ deviations in the up-type matrices that are responsible for the largest contributions to $\bar{\theta}$. We find that at sufficiently small $t_{0}$, the contributions to $\bar{\theta}$ are independent of $t_{0}$ and of the size

$$
\bar{\theta} \approx 10^{-13} \quad\left(\text { small } t_{0}\right)
$$

\subsection{Unitarity of $V_{\mathrm{CKM}}$}

Due to mixing between zero-modes and KK modes, the full CKM matrix restricted to zero modes is not exactly unitary. In models with UV-localized doublets, this effect is one to two orders of magnitude below the bound of current experimental constraints [22]. However, because of the larger mixing between zero-modes and KK-modes when doublets are on the $\mathrm{TeV}$ brane, this effect is much larger in our model. The strongest constraints on unitarity can be parameterized by $\Delta V_{i} \equiv 1-\sum_{j=1}^{3}\left|V_{i j}\right|^{2}$. The mixing is approximately $\mathcal{O}\left(\sqrt{2} v / m_{\mathrm{KKf}}\right)$ for the doublets, so

$$
\Delta V_{i} \lesssim \frac{2 v^{2}}{\left(m_{\mathrm{KKf}}^{(1)}\right)^{2}} \frac{\pi^{2}}{6}
$$

where the additional factor $\sum_{n} \frac{1}{n^{2}}=\pi^{2} / 6$ approximates the contribution from the tower of KK modes with masses $\sim m_{\mathrm{KKf}}^{(n)} \sim n m_{\mathrm{KKf}}^{(1)}$. This is at the level of current experimental constraints when $m_{\mathrm{KKf}}=9-12 \mathrm{TeV}$. This is comparable to though weaker than electroweak constraints on the model. 


\subsection{Flavor-changing neutral currents}

Strong constraints on physics beyond the Standard Model arise from dimension-6 flavorchanging operators:

$$
\mathcal{L}_{\Delta F=2} \supset \frac{z_{s d}}{\Lambda^{2}}\left(\bar{d}_{L} \gamma_{\mu} s_{L}\right)^{2}+\frac{z_{b d}}{\Lambda^{2}}\left(\bar{d}_{L} \gamma_{\mu} b_{L}\right)^{2}
$$

There are stronger constraints on the R-L operators such as $\left(s_{L} d_{R}\right)\left(d_{L} s_{R}\right)$. However, because of the small mixing in the right-handed fermions, this is very suppressed and the dominant constraints come from the above operators (see equation (B.12), which is nearly flavor-diagonal when restricted to zero modes). With $\Lambda \equiv \mathrm{TeV}$, the constraints on the $z$ coefficients are 23

$$
\begin{array}{rlrl}
\operatorname{Im}\left(z_{s d}\right) & \lesssim 6 \times 10^{-9} & \epsilon_{K} \\
z_{b d} & \lesssim 6 \times 10^{-6} & & \Delta m_{B}
\end{array}
$$

These operators get contributions from KK gluon exchange. The KK gluons, like all KK gauge bosons, are peaked at the $\mathrm{TeV}$ brane, with $\chi^{(i)}(1)=4.72 \approx \sqrt{2 k r_{c}}$. In the case of anarchic Yukawas with the left-handed fields localized on the $\mathrm{TeV}$ brane, the constraints from FCNCs would be much more severe. However, the situation is quite different with sequestered flavor and CP violation. When flavor-violation is sequestered, the quark-KK-gluon vertices become approximately flavor conserving in the mass eigenbasis. We have already worked out the form of the KK gluon interactions with the left-handed fermions in the mass eigenbasis in equation (B.14). Here, we are concerned with interactions with two zero mode fermions, which are described by the upper-left block of $(\overline{B .14}): L_{d}^{(0)}\left(\chi(1) \mathbf{1}+\frac{v}{m_{\mathrm{KK}}} D_{d L} \frac{v}{m_{\mathrm{KK}}}\right) L_{d}^{(0) \dagger}$. The matrix $L_{d}^{(0)}$ is not exactly unitary, so $L_{d}^{(0)} L_{d}^{(0) \dagger}$ has some off-diagonal components that contribute to $z_{s d}, z_{b d}$. We find numerically that, given our values for the $\nu$ 's and sampling over random $\Phi$ vevs, that $\left(L_{d}^{(0)} L_{d}^{(0) \dagger}\right)_{s d} \approx 4 V_{s d} \frac{\delta m}{m_{\mathrm{KK}}} \frac{v^{2}}{m_{\mathrm{KK}}^{2}}$, where $\delta m \approx 0.1 m_{\mathrm{KK}}$ is the small splitting in the KK fermion mass eigenvalues. Mixing between the first and third generation is even more suppressed: $\left(L_{d}^{(0)} L_{d}^{(0) \dagger}\right)_{b d} \approx 0.1 V_{b d} \frac{\delta m}{m_{\mathrm{KK}}} \frac{v^{2}}{m_{\mathrm{KK}}^{2}}$.

Gluon exchange involves two of the above interactions, so the contribution to $\left(\bar{b}_{L} \gamma_{\mu} d\right)^{2} / \Lambda^{2}$ in the small $t_{0}$ limit is approximately ${ }^{8}$

$$
\begin{aligned}
z_{b d} & \approx(1.4)\left(2 k \pi r_{c}\right) g_{s}^{2} \frac{\left[\lambda_{C}^{3} 0.1\left(\delta m / m_{\mathrm{KKf}}\right)\left(v / m_{\mathrm{KKf}}\right)^{2}\right]}{m_{K K g}^{2}} \\
& \approx 6 \times 10^{-6}\left(\frac{400 \mathrm{GeV}}{m_{\mathrm{KKg}}}\right)^{6}
\end{aligned}
$$

where the 1.4 has been included to account for contributions from higher KK modes.

\footnotetext{
${ }^{8} \mathrm{We}$ assume $m_{\mathrm{KKg}} \approx \frac{2.45}{\pi} m_{\mathrm{KKf}}$, which typically follows from the equations of motion for KK gauge bosons and KK fermions in RS models.
} 
The constraints from $\epsilon_{K}$ are significantly more severe:

$$
\begin{aligned}
\operatorname{Im}\left(z_{s d}\right) & \approx(1.4)\left(2 k \pi r_{c}\right) g_{s}^{2} \frac{\left[\lambda_{C} 4\left(\delta m / m_{\mathrm{KKf}}\right)\left(v / m_{\mathrm{KKf}}\right)^{2}\right]}{m_{K K g}^{2}} \\
& \approx 9 \times 10^{-6}\left(\frac{10 \mathrm{TeV}}{m_{K K g}}\right)^{6}
\end{aligned}
$$

\section{Conclusion}

We have considered a new solution to the strong $\mathrm{CP}$ problem based on spontaneous $\mathrm{CP}$ violation in RS models. We sequester the source of $\mathrm{CP}$ violation, which we assume results from the vev of a scalar field localized near the UV brane, from the Yukawa interactions in the IR with the Higgs, which we assume respect a large flavor symmetry. In order to suppress radiative contributions to $\bar{\theta}$, we localize the doublet quarks on the IR brane, which pushes the scale of $\mathrm{KK}$ gauge bosons up to be $\gtrsim 15-18 \mathrm{TeV}$. We find $\bar{\theta}$ suppressed to as little as $10^{-13}$.

Let us contrast our model with other models of spontaneous CPV, beginning with the $4 \mathrm{D}$ dual of our warped scenario. Interestingly, the mechanism that enforces a vanishing strong CP phase at tree level in the gravitational theory is slightly different from in the CFT dual. In 5D the hermiticity of bulk fermion mass matrices is enforced by higher dimensional spacetime symmetries. Where the dual is conformal (that is, excluding energies near CFT breaking), these masses correspond to anomalous dimensions, which must be hermitian simply because they are renormalizations of the kinetic terms.

Let us consider the structure of the CFT as we flow down from high energies. In the $\mathrm{UV}, \mathrm{CP}$ is a valid symmetry and the theory is conformal. At this scale, the UV localized SM fermions are present in vector-like pairs and as fundamental objects. At the scale $\Lambda_{C P V}$ the CFT strongly couples, generating a bound state scalar CP-on $\Phi$ which spontaneously breaks CP. At this point conformal symmetry is maximally violated by strong dynamics, so we would naturally assume that $\mathrm{CP}$ phases enter the theory quite generically. However, as we can compute in the gravity theory, this is not the case, and strong dynamics introduces only (hermitian) wavefunction renormalizations of the (now partly composite) fermions. Below this scale, the residual CFT gauge symmetry flows down to the IR brane, where it strongly couples again, yielding a composite Higgs scalar and fermion composites corresponding to the heavier SM fermions. In general, the $4 \mathrm{D}$ dual of our model works by keeping all phases inside wavefunction renormalizations.

This is similar to what we will call theories of "wavefunction renormalization." These models utilize spontaneous CP violation, but subject to symmetries that effectively sequester CP phases in wavefunction renormalizations (or higher-dimensional wavefunctions) alone. Because any renormalization of the kinetic energy term necessarily implies that the anomalous dimensions are hermitian, these models forbid strong CP phases at tree level.

For example, in Hiller-Schmaltz [28], the supersymmetric non-renormalization theorem is used to forbid CP phases from seeping into $\bar{\theta}$, which is only in the superpotential. Because CP violation occurs above the SUSY breaking scale, phases enter only into the 
Kahler potential, and are thus hermitian up to SUSY-breaking effects. However, in order to obtain appropriately large weak CP phases, these wavefunction renormalizations must be of order unity, and so this theory is strongly coupled. As a result, precise model-dependent observables are difficult to obtain, and moreover the inclusion of higher dimension operators generated by these strong dynamics force the $\mathrm{CP}$ violating scale up to around $10^{5} \mathrm{GeV}$, far above future collider reach. Because our model is gravitational, it is completely weakly coupled. Otherwise, the 4D CFT dual of our theory has many similarities to Hiller-Schmaltz, except that conformal symmetry effectively replaces supersymmetry in terms of suppressing phases in the potential.

Our model also has advantages over alternative models of spontaneous CP violation. For example, consider the archetypal model of spontaneous CP violation, proposed by Nelson and Barr [26, 27]. This model assumes an exact CP symmetry in the UV, as well as additional scalars and vector-like fermions at a high scale charged under the SM flavor group. After a scalar gets a $\mathrm{CP}$ violating vev, $\bar{\theta}$ is forbidden at tree-level due to conditions placed on the mass matrix by the GUT representations of additional fermions.

From a model-building point of view, our proposal can be seen as more minimal than Nelson-Barr. First of all, while the inclusion of vector-like fermions charged under added flavor symmetries is not necessary in a 4D model, it follows from the KK reduction in 5D. In particular, 5D spacetime symmetries necessarily imply that parity is a symmetry of the bulk, forcing heavy modes to appear in vector-like pairs. Furthermore, the mass of the new vector-like states can be much lower. Finally, we note that since our model is in RS, we can explain large hierarchies of mass scales through the warped geometry.

\section{Acknowledgments}

We would like to acknowledge helpful conversations with Patrick Meade, Jared Kaplan, Matthew Schwartz, Patrick Fox, and Nima Arkani-Hamed, and Gilad Perez for conversations and comments on the draft. ALF would like to thank Matthew Baumgart for several clarifying discussions of electroweak precision constraints. LR is supported in part by NSF grants PHY-0201124 and PHY-0556111. ALF is supported by an NSF Graduate Research Fellowship. CC is supported in part by DOE grant DE-FG02-91ER40654. Any opinions, findings, and conclusions or recommendations expressed in this material are those of the authors and do not necessarily reflect the views of the National Science Foundation.

\section{A. Bulk fermion wavefunctions}

\section{A.1 Wavefunctions without twisting}

In this subsection we derive bulk fermion wavefunctions in a simply warped model with no flavor twisting. A right-handed fermion in RS with bulk mass $m=\nu k$ satisfies the wave equation

$$
\left(\partial_{t}^{2}+x^{2}-\frac{\nu(\nu+1)}{t^{2}}\right) f_{R}=0
$$


The solutions are

$$
f_{R}(t)=\sqrt{t}\left(\beta J_{\frac{1}{2}+\nu}(x t)-\alpha J_{-\frac{1}{2}-\nu}(x t)\right)
$$

The three conditions that completely determine $\alpha, \beta$, and $x$ are the two boundary conditions, $f_{R}(\epsilon), f_{R}(1)=0$, and the normalization $\int_{\epsilon}^{1} f_{R}^{2}(t) d t=1$. It is useful to work out approximate solutions first. The $t=\epsilon$ condition is, to lowest-order in $\epsilon$,

$$
0=-\frac{2^{\frac{1}{2}+\nu} x^{-\frac{1}{2}-\nu} \alpha \epsilon^{-\nu}}{\Gamma\left(\frac{1}{2}-\nu\right)}+\frac{2^{-\frac{1}{2}-\nu} x^{\frac{1}{2}+\nu} \beta \epsilon^{1+\nu}}{\Gamma\left(\frac{3}{2}+\nu\right)}
$$

so $\alpha \ll \beta$ if $\nu>-\frac{1}{2}$, and $\alpha \gg \beta$ if $\nu<-\frac{1}{2}$. The boundary condition at $t=1$ is therefore

$$
0 \approx\left\{\begin{array}{c}
J_{-\frac{1}{2}-\nu}(x), \nu<-\frac{1}{2} \\
J_{\frac{1}{2}+\nu}(x), \quad \nu>-\frac{1}{2}
\end{array}\right.
$$

The $n$-th root of $J_{\mu}$ is well-approximated by $\left(\frac{\mu}{2}-\frac{1}{4}+n\right) \pi$ in the range $5>\mu>-1 / 2$. Over the range $-1<x<1$, this approximates $x$ to within $5 \%$. So, we can take

$$
x^{(n)} \approx\left\{\begin{array}{cc}
\left(\frac{\nu}{2}+n\right) \pi, \quad \nu>-\frac{1}{2} \\
\left(-\frac{\nu}{2}-\frac{1}{2}+n\right) \pi, \quad \nu<-\frac{1}{2}
\end{array}\right.
$$

It is convenient to take $\mu=\left|\nu+\frac{1}{2}\right|$. Continuing to take $\epsilon \ll 1$, the normalization condition for $\nu>-\frac{1}{2}$ is

$$
1=\int_{\epsilon}^{1} f_{R}^{2}(t) d t=\frac{\beta^{2}}{2} J_{\mu+1}^{2}(x)
$$

Thus, we have approximately

$$
f_{R}(t)=\left\{\begin{array}{l}
\frac{\sqrt{2 t} J_{\frac{1}{2}+\nu}(x t)}{J_{-\frac{1}{2}+\nu}(x)}, \nu>-\frac{1}{2} \\
\frac{\sqrt{2 t} J_{-\frac{1}{2}-\nu}(x t)}{J_{\frac{1}{2}-\nu}(x)}, \nu<-\frac{1}{2}
\end{array}\right.
$$

where $x$ satisfy equation (A.4). In terms of the coefficients $\alpha, \beta$, this is

$$
\begin{array}{ll}
\nu>-\frac{1}{2}: \alpha=0, & \beta=\frac{\sqrt{2}}{J_{\left|\nu-\frac{1}{2}\right|}(x)} \\
\nu<-\frac{1}{2}: \alpha=\frac{\sqrt{2}}{J_{\left|\nu-\frac{1}{2}\right|}(x)}, & \beta=0
\end{array}
$$

Thus, the left-handed excited mode wavefunctions are given by

$$
f_{L}(t)=\left\{\begin{array}{c}
\frac{\sqrt{2 t} J_{-\frac{1}{2}+\nu}(x t)}{J_{-\frac{1}{2}+\nu}(x)}, \nu>-\frac{1}{2} \\
-\frac{\sqrt{2 t} J_{\frac{1}{2}-\nu}(x t)}{J_{\frac{1}{2}-\nu}(x)}, \nu<-\frac{1}{2}
\end{array}\right.
$$

and the zero mode is:

$$
f_{L}^{0}=\sqrt{\frac{1+2 \nu}{1-\epsilon^{1+2 \nu}}} t^{\nu}
$$




\section{A.2 Wavefunctions with twisting}

Solving for the wavefunctions can be complicated by twisting. However, because of the delta function form of $\Phi$, the bulk fermion wavefunctions can be straightforwardly solved for on to the left and right of $\phi_{0}$, and then matched at the junction.

We now show this explicitly. The following discussion will hold equally well for $U$ and $D$, so we will drop all $u, d$ subscripts and let $X=g r\langle\Phi\rangle$. Rewriting in terms of the $t$ variable, we find that $\delta\left(\phi-\phi_{0}\right)=k r t \delta\left(t-t_{0}\right)$, and so from eq. (3.12) we can write $\mu=\operatorname{diag}\left(\nu_{i}\right)+X t_{0} \delta\left(t-t_{0}\right)$. where $\nu_{i}$ ultimately sets the masses of the bulk fermions. Eq. (3.11) depicts the first order wave equations for a bulk fermion written as a matrix differential operator acting on a matrix wavefunction. For computational simplicity, let us consider a single column of this equation, which constitutes a matrix differential operator acting on a vector wavefunction:

$$
\begin{aligned}
\left( \pm \partial_{t}-\frac{\mu}{t}\right) f_{L, R} & =-x f_{R, L}, \\
\Rightarrow\left(-\partial_{t}^{2}+\frac{\mu(\mu \mp 1) \pm t \mu^{\prime}}{t^{2}}\right) f_{L, R} & =x^{2} f_{L, R},
\end{aligned}
$$

where $f_{L, R}$ are 3 -vectors, ' denotes differentiation with respect to $t$, and $x$ is a mass eigenvalue which will be determined by the orbifold boundary conditions on the wavefunctions.

The second order equation in eq. (A.10) has a well-known solution in terms of Bessel functions,

$$
\begin{aligned}
f_{L} & =J_{+-}(t) a_{L}+J_{-+}(t) a_{R}, \\
f_{R} & =J_{++}(t) a_{R}-J_{--}(t) a_{L}, \\
J_{ \pm \pm}(t) & =\sqrt{t} \operatorname{diag}\left(J_{ \pm \frac{1}{2} \pm \nu_{i}}(x t)\right),
\end{aligned}
$$

where the $a_{L, R}$ are constant 3 vectors defined piece-wise by

$$
a_{L, R}=\left\{\begin{array}{l}
a_{L, R}^{+} t>t_{0} \\
a_{L, R}^{-} t<t_{0}
\end{array}\right.
$$

As written, there are precisely four vectors $\left(a_{L}^{+}, a_{R}^{+}, a_{L}^{-}\right.$and $\left.a_{R}^{-}\right)$of initial conditions to fix. Since one of these is an overall normalization, this leaves three vector unknowns.

Now if we take $f_{R}$ to be odd under the orbifold symmetry, then it vanishes at the orbifold fixed points,

$$
f_{R}(\epsilon)=f_{R}(1)=0
$$

These two boundary conditions, along with the zeroth and first derivative boundary conditions at $t_{0}$ constitute four boundary conditions that will act to (over-)constrain the three initial conditions for the fermion wavefunctions. Since this over-constrains the system, this puts a constraint on $x$ and fixes the allowed masses to a discretum. Enforcing equation (A.15) allows us to eliminate the $a_{L}$ 's and write

$$
f_{R}(t)=\left\{\begin{array}{l}
\left(\frac{J_{--}(1) J_{++}(t)-J_{++}(1) J_{--}(t)}{J_{--}(1)}\right) a_{R}^{+} t>t_{0} \\
\left(\frac{J_{++}(t) J_{--}(\epsilon)-J_{--}(t) J_{++}(\epsilon)}{J_{--}(\epsilon)}\right) a_{R}^{-} t<t_{0}
\end{array}\right.
$$


Next, let determine the matching conditions at the third brane. Because the first order equation in eq. A.10 relates the first derivative of the fermion wavefunction to a delta function, we know that there is jump discontinuity at $t_{0}$. Moreover, without loss of generality, we can regulate this jump with a linear interpolating function, so

$$
f_{L, R}\left(t_{0}\right)=\frac{1}{2}\left(f_{L, R}\left(t_{0}^{+}\right)+f_{L, R}\left(t_{0}^{-}\right)\right),
$$

where \pm denote limiting values from the right and left. Our solutions will of course be insensitive to this regulation.

The first boundary condition is then easily obtained by integrating the first order wave equation over a small neighborhood around $t_{0}$ :

$$
-\left.f_{R}\right|_{t_{0}^{-}} ^{t_{0}^{+}}-\int_{t_{0}^{-}}^{t_{0}^{+}} \frac{\mu}{t} f_{R} d t=-x \int_{t_{0}^{-}}^{t_{0}^{+}} f_{R} d t .
$$

Since the integrand on the r.h.s. is not singular at $t_{0}$ except for a discontinuity and it is integrated over an infinitesimal range, its contribution vanishes. In fact only the delta function component of $\mu$ survives the integration, yielding

$$
\begin{aligned}
f_{R}\left(t_{0}^{+}\right)-f_{R}\left(t_{0}^{-}\right) & =-\frac{1}{2} X\left(f_{R}\left(t_{0}^{+}\right)+f_{R}\left(t_{0}^{-}\right)\right), \\
\Rightarrow f_{R}\left(t_{0}^{+}\right) & =R f_{R}\left(t_{0}^{-}\right), \\
R & =\frac{1-X / 2}{1+X / 2} \\
& \approx 1-X
\end{aligned}
$$

where the last relation holds in the limit that $X$ is small.

Next, we find the second boundary condition at $t_{0}$ by integrating the second order wave equation over an infinitesimal neighborhood around $t_{0}$ :

$$
-\left.f_{R}^{\prime}\right|_{t_{0}^{-}} ^{t_{0}^{+}}+\int_{t_{0}^{-}}^{t_{0}^{+}} \frac{\mu(\mu \mp 1) \pm t \mu^{\prime}}{t^{2}} f_{R} d t=x^{2} \int_{t_{0}^{-}}^{t_{0}^{+}} f_{R} d t .
$$

Plugging in for $\nu$ we find that

$$
\left.f_{R}^{\prime}\right|_{t_{0}^{-}} ^{t^{+}} \pm X f_{R}^{\prime}\left(t_{0}\right)-\frac{1}{t_{0}}\left(\left\{\operatorname{diag}\left(\nu_{i}\right), X\right\}+X t_{0} \delta(0)\right) f_{R}\left(t_{0}\right)=0 .
$$

Here we have introduced a $\delta(0)$ which can be easily removed by plugging in the first order wave equation evaluated at $t_{0}$ :

$$
-f_{R}^{\prime}\left(t_{0}\right)-\frac{1}{t_{0}}\left(\operatorname{diag}\left(\nu_{i}\right)+X t_{0} \delta(0)\right) f_{R}\left(t_{0}\right)=-x f_{L}\left(t_{0}\right)
$$

Combining these equations and plugging in for $f_{L}\left(t_{0}\right)$ in terms of $f_{R}\left(t_{0}^{+}\right)$and $f_{R}\left(t_{0}^{-}\right)$from the first order wave equation, we finally obtain the two boundary conditions for the fermion 
wavefunctions at $t_{0}$ :

$$
\begin{aligned}
f_{R}\left(t_{0}^{+}\right) & =R f_{R}\left(t_{0}^{-}\right) \\
f_{R}^{\prime}\left(t_{0}^{+}\right) & =R^{-1} f_{R}^{\prime}\left(t_{0}^{-}\right)+S f_{R}\left(t_{0}^{-}\right) \\
R & =\frac{1-X / 2}{1+X / 2} \\
S & =\frac{1}{1-X / 2} \frac{\left\{\operatorname{diag}\left(\nu_{i}\right), X\right\}}{t_{0}} \frac{1}{1+X / 2} .
\end{aligned}
$$

The above equations, along with the two orbifold boundary conditions constitute four linear matrix equations which can be combined (after plugging in $f_{L, R}$ ) in order to yield an equation relating $a_{R}^{+}$and $a_{R}^{-}$

$$
a_{R}^{+}=\frac{J_{--}(1)}{J(1)} R \frac{J(\epsilon)}{J_{--}(\epsilon)} a_{R}^{-}
$$

and a single condition on $a_{R}^{+}$:

$$
G(x) a_{R}^{+}=0
$$

where we have defined the matrix functions

$$
\begin{aligned}
J(t) & \equiv J_{--}(t) J_{++}\left(t_{0}\right)-J_{++}(t) J_{--}\left(t_{0}\right) \\
G(x) & \equiv\left(-\partial_{t_{0}} J(1) R J(\epsilon)+J(1) R^{-1} \partial_{t_{0}} J(\epsilon)+J(1) S J(\epsilon)\right) J_{--}^{-1}(\epsilon) .
\end{aligned}
$$

The matrix $G(x)$ does not have zeros for any arbitrary $x$, and so this linear equation forces $x$ to take on a discretum of values. It is straightforward to numerically obtain the zeros of $\operatorname{det} G(x)$, thus yielding the fermion mass eigenvalues $x_{i}$. Then, the $a_{R}^{+}$corresponding to the mass eigenvalue $x_{i}$ is the null eigenvector of $G\left(x_{i}\right)$, and $a_{R}^{-}$is given by equation (A.30). Thus from equation (A.16) we have the fermion bulk wave functions.

\section{B. Analysis of flavor spurions in small $t_{0}$ limit}

To begin, we go to mass eigenbasis, which is advantageous because this separates out the flavor-mixing, which occurs only at interaction vertices, from other sources of flavorbreaking. We consider the (broken) chiral flavor symmetry $G_{F}=\left(\mathrm{SU}(3)_{L} \times \mathrm{SU}(3)_{R}\right)^{N_{\mathrm{KK}}}$ which acts separately on each KK level, treating the symmetry-breaking terms as spurions charged under this symmetry. Also, let us first consider diagrams with only neutral current interactions, and generalize our argument later to include both charged and neutral currents. Thus for the present, loop diagrams necessarily contain only up-like or only down-like quarks, and we can first consider only with the part of $G_{F}$ which acts on the down-like quarks. We will consider a general element of $G_{F}, Z_{L} \times Z_{R} \times K_{L} \times K_{R}$, where $Z_{L}\left(Z_{R}\right)$ is a special unitary transformation that acts on the left-handed (right-handed) zero modes and $K_{L}\left(K_{R}\right)$ is a special unitary transformation that act on the first excited KK left-handed (right-handed) modes. Since the mass matrix, $L_{d}, R_{d}, C_{d R}$, and $C_{d L}$ all 
break this symmetry, we treat their zero mode and KK blocks as spurions. We will now describe these interactions in some detail in the small $t_{0}$ limit and catalogue the spurions under the broken flavor symmetry $G_{F}$.

To begin, consider the interactions between fermions and gluons before KK reducing:

$$
\mathcal{L} \supset g_{3} \sqrt{\pi} A_{\mu}\left(\bar{q}_{i} \gamma^{\mu} q_{i} \delta(t-1)+\bar{U}_{i} \gamma^{\mu} U_{i}+\bar{D}_{i} \gamma^{\mu} D_{i}\right)
$$

Let us denote the wave-function for the $(n)$-th KK gauge boson by $\chi_{A}^{(n)}(t)$, in a convention where

$$
A_{\mu}(x, t)=\sum_{n=0}^{N} A_{\mu}^{(n)} \frac{\chi_{A}^{(n)}(t)}{\sqrt{r_{c}}}
$$

In this convention, the zero mode wavefunctions $\chi_{A}^{(0)}(t)=1 / \sqrt{\pi}$ are flat, the excited mode wavefunctions satisfy $\chi_{A}^{(n)}(1) \approx \sqrt{2 k r_{c}}$ where $n>0$. Because the zero mode wavefunction is a constant and the fermion KK modes are orthonormal, the interactions with the zero mode gauge boson are exactly flavor-respecting. This is of course the result of the exact gauge symmetry.

As it turns out, the KK gluon interactions also possess an approximate flavor symmetry. To see this, consider the coupling of the $(n)$-th KK gluon to the zero mode and first KK mode fermions. We will write this interaction in terms of the $u_{L, R}$ basis defined in equations (4.5)-(4.9), where the approximate $\mathrm{U}(1)^{3}$ flavor symmetry is respected by the KK mode masses. We refer to this basis as the "KK basis", to distinguish it from the mass eigenbasis where the full mass matrix including mixing with the zero modes and KK modes is diagonal.

In the KK basis, the gluon coupling to the $u_{R}$ is given by the integral of the KK wavefunctions over the fifth dimension:

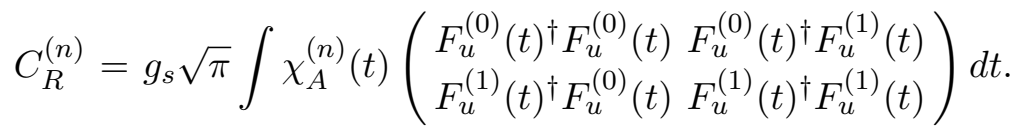

Here each $F$ is a three by three flavor matrix. For the case of $u_{L}$, the gluon interaction matrix is simpler since the doublet fields have no KK excitations:

$$
C_{L}^{(n)}=g_{s} \sqrt{\pi} \int \chi_{A}^{(n)}(t)\left(\begin{array}{cc}
\delta(t-1) & 0 \\
0 & F_{u^{\prime}}^{(1)}(t)^{\dagger} F_{u^{\prime}}^{(1)}(t)
\end{array}\right) d t
$$

where $F_{u^{\prime}}$ denotes the bulk wavefunction corresponding to the left handed KK excitation of the right handed singlet $u_{R}^{(0)}$.

The coupling of the fermions to a KK gluon can be understood qualitatively by decomposing the gluon wavefunction into a flat "UV" piece that is constant and an oscillating "IR" piece that vanishes in the UV:

$$
\begin{aligned}
& \chi_{A}^{(n)}(t)=\chi_{\mathrm{UV}}(t)+\chi_{\mathrm{IR}}^{(n)}(t) \\
& \chi_{\mathrm{UV}}(t)=-\frac{1}{\sqrt{8 k r_{c}}} \\
& \chi_{\mathrm{IR}}^{(n)}(t) \approx \sqrt{2 k r_{c} t} \sin \left(t \frac{2 n-1}{2} \pi\right)
\end{aligned}
$$


The "UV" piece is flat and therefore, by orthonormality of the fermion wavefunctions, flavor-universal. For $C_{R}^{(n)}$, the IR contribution roughly yields products of $F_{u}$ 's evaluated near the IR brane, and thus has the same order of magnitude as the Yukawa matrices. We thus obtain qualitatively

$$
C_{R}^{(n)} \approx g_{s} \sqrt{\pi}\left(-\frac{1}{\sqrt{8 k r}} \mathbf{1}+\sqrt{2 k r} Y_{u}^{\dagger} Y_{u}\right)
$$

This implies that the KK gluon vertices that mix right-handed zero modes fermions and right-handed KK mode fermions are suppressed by the zero mode masses, which we will find later to be essential to making radiative corrections to $\bar{\theta}$ small.

The left-handed partners of the KK singlets have wavefunctions that vanish on the IR brane, and their coupling to the KK gluon does not receive the full $\sqrt{2 k r_{c}}$ volume factor:

$$
C_{L}^{(n)} \approx g_{s} \sqrt{\pi}\left(\begin{array}{cc}
\sqrt{2 k r} \mathbf{1} & 0 \\
0 & \mathcal{O}(2)
\end{array}\right) .
$$

For radiative corrections to $\bar{\theta}$ to be small, we need small $t_{0}$. We can describe the interactions approximated in equations (B.8) and (B.9) more precisely in this small $t_{0}$ limit, where the KK mode fermion wavefunctions are independent of $\Phi$ but the zero mode fermion wavefunctions are not. The essential point will be that the only sources of flavor mixing are the left-handed diagonalization matrices $L_{u, d}^{(0)}$ for the zero modes and that the only new flavor-violating spurions beyond those in the Standard Model that can contract with $L_{u, d}^{(0)}$ are suppressed by $\left(v / m_{K K f}\right)^{2}$ or the usual mass matrix for the zero modes. Thus, we now wish to demonstrate that all flavor-mixing in the gluon vertices can be pushed in the mass eigenbasis into $L_{u}^{(0)}$ and $L_{d}^{(0)}$.

First, we show that $L_{u, d}^{(0)}$ are also the only sources of flavor mixing in the gluon interaction vertices. In particular, $R_{u, d}^{(0)}$ can be absorbed into the diagonalizations of the zero mode masses (and equivalently $F_{u, d}^{(0)}$ ) so that they manifestly do not contribute new sources of flavor-mixing. We will restrict our attention to the gluon interaction with down quarks for the moment, to avoid writing extra indices. First, note that since the KK fermion wavefunctions are diagonal in the present limit, their interactions with the KK gauge boson are also diagonal in the KK basis. We can denote this by defining $D_{d R}=C_{d R, 11}$, so $D_{d R}$ is a diagonal matrix. Second, consider the interactions of the KK gauge bosons with a zero mode fermion and a KK mode fermion. These are given by $C_{d R, 01}=g_{s} \sqrt{\pi} \int_{\epsilon}^{1} \chi(t) F_{d}^{(0) \dagger}(t) F_{d}^{(1)}(t)$. However, the integral receives only a negligible contribution from $t<t_{0}$, and above that $F_{d}^{(0) \dagger}(t)=F_{d}^{(0) \dagger}\left(t_{0}\right)$ times a diagonal matrix, according to equation (3.16). This implies that at any point $t>t_{0}, F_{d}^{(0) \dagger}(t)$ is related to $F_{d}^{(0) \dagger}(1)$ by a diagonal matrix. Thus, $C_{d R, 01}=F_{d}^{(0) \dagger}(1) D_{d R}^{\prime}$, where we have defined another diagonal matrix $D_{d R}^{\prime}$. Finally, note that because the zero modes are UV localized and do not oscillate in the IR, they see the IR piece $\chi_{\mathrm{IR}}$ of the KK gluon wavefunction as essentially a spike near the IR brane, and thus the qualitative approximation of $C_{d R}$ we gave earlier is quantitatively very good for the zero modes: $C_{d R, 00} \approx g_{s} \sqrt{\pi}\left(-\frac{1}{\sqrt{8 k r_{c}}} \mathbf{1}+\sqrt{2 k r_{c}} F_{d}^{(0) \dagger} F_{d}^{(0)}\right)$. Furthermore, the effect of deviations from this approximation are suppressed by the zero mode wavefunctions. 
The exact interaction contains the flavor-violating contribution $F_{d}^{(0) \dagger} D_{d R ; 0} F_{d}^{(0)}$, again with $D_{d R ; 0}$ a diagonal matrix. In the mass eigenbasis, this is $f_{d}^{(0)} L_{d}^{(0)} D_{d R ; 0} L_{d}^{(0) \dagger} f_{d}^{(0)}$. The small off-diagonal pieces of $L_{d}^{(0)} D_{d R ; 0} L_{d}^{(0) \dagger}$ are then further suppressed by the hierarchy in zero mode masses, making them completely negligible. Thus, in the KK basis, $C_{d R}$ takes the form

$$
C_{d R}=g_{s} \sqrt{\pi}\left(\begin{array}{cc}
\chi(\epsilon) \mathbf{1}+\chi(1) F_{d}^{(0) \dagger} D_{d R ; 0} F_{d}^{(0)} & F_{d}^{(0) \dagger} D_{d R}^{\prime} \\
D_{d R}^{\prime} F_{d}^{(0)} & D_{d R}
\end{array}\right)
$$

Consider now the rotation to mass eigenbasis. The right-handed mixing between zero modes and KK modes is suppressed by zero mode masses and $v / m_{\mathrm{KKf}}$, where we find numerically the result

$$
R_{d}=\left(\begin{array}{cr}
R_{d}^{(0)} & -R_{d}^{(0)} F_{d}^{(0) \dagger} \frac{\sqrt{2} v^{2}}{m_{\mathrm{KKf}}^{2}} \\
\frac{\sqrt{2} v^{2}}{m_{\mathrm{KKf}}^{2}} F_{d}^{(0)} & \mathbf{1}
\end{array}\right)
$$

We now use this to rotate $C_{d R}$ to the mass eigenbasis:

$$
C_{d R} \rightarrow\left(\begin{array}{cc}
\chi(\epsilon) \mathbf{1}+\chi(1) f_{d}^{(0) 2} & f_{d}^{(0)} L_{d}^{(0)} D_{d R}^{\prime} \\
D_{d R}^{\prime} L_{d}^{(0) \dagger} f_{d}^{(0)} & D_{d R}
\end{array}\right)+\mathcal{O}\left(\frac{v^{2} f_{d}^{(0) 2}}{m_{K K f}^{2}}\right)
$$

We have not written out some terms that are higher order in $\frac{v^{2}}{m_{\mathrm{KK}}^{2}}$. This is because they can be written as spurions we have already included (times some diagonal matrices) and thus can only give contributions that are suppressed by $v^{2} / m_{\mathrm{KK}}^{2}$ over those we are including. The above form demonstrates our claim that $R_{d}^{(0)}$ does not explicitly appear in the gluon interactions, and thus the only source of flavor-mixing in the down-type interactions is $L_{d}^{(0)}$. The matrix of KK gauge boson interactions with the left-handed fields is simpler. In the KK basis, it takes the form we have already derived:

$$
C_{d L}=\left(\begin{array}{cc}
\chi(1) 1 & 0 \\
0 & D_{d L}
\end{array}\right)
$$

and thus in the mass eigenbasis does not contain any new flavor-breaking spurions beyond $D_{d L}$ and those in $L_{d}$. Explicitly, it transforms to

$$
C_{d L} \rightarrow\left(\begin{array}{cc}
L_{d}^{(0)}\left(\chi(1) \mathbf{1}+\frac{v}{m_{\mathrm{KK}}} D_{d L} \frac{v}{m_{\mathrm{KK}}}\right) L_{d}^{(0) \dagger} & L_{d}^{(0)} \frac{v}{m_{\mathrm{KK}}}\left(\chi(1) \mathbf{1}-D_{d L}\right) \\
\left(\chi(1) \mathbf{1}-D_{d L}\right) \frac{v}{m_{\mathrm{KK}}} L_{d}^{(0) \dagger} & \chi(1)\left(\frac{v}{m_{\mathrm{KK}}^{2}}+D_{d L}\right)
\end{array}\right)
$$

In particular, the mass matrix supplies the three spurions $f_{d}^{(0)}, \sqrt{2} \mathbf{1}$, and $M^{(1)}, L_{d}$ supplies the spurions $L_{d}^{(0)}$ and $\frac{v}{m_{\mathrm{KKF}}}$, and $C_{d R}$ and $C_{d L}$ supply the spurions $F_{d}^{(0) \dagger} D_{d R}^{\prime}, D_{d R}$, 
and $D_{d L}$. Under the chiral flavor symmetry, these spurions transform like

$$
\begin{aligned}
f_{d}^{(0)} & \rightarrow Z_{L} F_{d}^{(0)} Z_{R}^{\dagger} \\
\sqrt{2} \mathbf{1} & \rightarrow Z_{L} \sqrt{2} K_{R}^{\dagger} \\
f_{d}^{(0)} L_{d}^{(0)} D_{d R}^{\prime} & \rightarrow Z_{R} F_{d}^{(0) \dagger} D_{d R}^{\prime} K_{R}^{\dagger} \\
D_{d L} & \rightarrow K_{L} D_{d L} K_{L}^{\dagger} \\
M_{1} & \rightarrow K_{L} M_{1} K_{R}^{\dagger} \\
L_{d}^{(0)} & \rightarrow Z_{L} L_{d}^{(0)} Z_{L}^{\dagger} \\
\frac{v}{m_{\mathrm{KKf}}} \mathbf{1} & \rightarrow Z_{L} \frac{v}{m_{\mathrm{KKf}}} \mathbf{1} K_{L}^{\dagger}
\end{aligned}
$$

Next, we estimate the value of $\bar{\theta}$ from a two loop diagram with neutral currents. First, we note that in order to obtain a contribution to $\bar{\theta}$, a diagram must necessarily include four factors of $L_{d}^{(0)}$. Otherwise the phase in the first flavor-mixing $L_{d i j}^{(0)}$ is precisely canceled by mixing back through the inverse factor $L_{d j i}^{(0) \dagger}$. The flavor symmetry then fixes which spurions can interleave around these four factors.

The only spurions that transform under $Z_{L}$ aside from $L_{d}^{(0)}$ are $f_{d}^{(0)}$ and $\frac{v}{m_{\mathrm{KKf}}}$. The first of these transforms on the right under $Z_{R}$, so it must be squared in order to appear between two $L_{d}^{(0)}$ 's. Note that these spurions are nothing more than the zero mode masses, and it is exactly this argument that implies such a large suppression of $\bar{\theta}$ in the Standard Model.

The second of these, $\frac{v}{m_{\mathrm{KKf}}}$, transforms on the right under $K_{L}$, so it also must be squared in order to appear between $L_{d}^{(0)}$ 's. Thus, it also gives a suppression comparable to $\left(m_{b} / v\right)^{2}$.

Next, if the external mode is a zero mode, then at least one of the spurions must be proportional to $f_{d}^{(0)}$ (to see this explicitly, note that all of the spurions charged under $Z_{R}$ are proportional to $f_{d}^{(0)}$ ), which then cancels with the $m_{d}^{-1}$ in the expression for corrections to $\bar{\theta}$. Thus, the minimum suppression allowed by the above argument is $\left(v / m_{\mathrm{KKf}}\right)^{7}=3 \times 10^{-12}$. Finally, there is the fact that the overall contribution must be proportional to the Standard Model phase invariant $\Delta^{(4)} \equiv \operatorname{Im}\left(L_{d 12}^{(0)} L_{d 22}^{(0) \dagger} L_{d 21}^{(0)} L_{d 11}^{(0) \dagger}\right) \sim 10^{-4}$ (see section (3.4)). This puts the final contributions from two neutral currents (or more specifically, two gluons) safely below the experimental limits on $\bar{\theta}$. We note that, when all of the loops are from KK gauge bosons, each vertex contains a large coupling $g_{5} \chi(1)=g_{4} \sqrt{2 k \pi r_{c}} \lesssim \sqrt{70}$, which is very close to the non-perturbative regime. In particular, each additional loop is suppressed only by a factor of $g_{4}^{2} \frac{2 k \pi r_{c}}{(4 \pi)^{2}}$.

We can now extend the above argument to loops including charged currents. In this case, we must consider a separate flavor symmetry acting on up-type quarks and downtype quarks: $G_{F}=G_{F u} \times G_{F d} \equiv\left(\mathrm{SU}(3)_{L} \times \mathrm{SU}(3)_{R}\right)_{u}^{N_{\mathrm{KK}}} \times\left(\mathrm{SU}(3)_{L} \times \mathrm{SU}(3)_{R}\right)_{d}^{N_{\mathrm{KK}}}$, since charged currents will generate diagrams with both up-like and down-like quarks. It is crucial that the only new flavor-mixing spurions from charged currents arise from $V_{\mathrm{CKM}}=$ 
$L_{u} L_{d}^{\dagger}$, which in the small $t_{0}$ limit takes the form

$$
V_{\mathrm{CKM}}=\left(\begin{array}{cc}
L_{u}^{(0)} L_{d}^{(0) \dagger} & L_{u}^{(0)} \frac{v}{m_{\mathrm{KKf}}} \\
\frac{v}{m_{\mathrm{KKf}}} L_{d}^{(0) \dagger} & \frac{v^{2}}{m_{\mathrm{KKf}}^{2}}
\end{array}\right)
$$

These new spurions therefore transform like:

$$
\begin{aligned}
V_{\mathrm{CKM}}^{(0)} \equiv L_{u}^{(0)} L_{d}^{(0) \dagger} & \rightarrow Z_{L}^{u} V_{\mathrm{CKM}}^{(0)} Z_{L}^{d \dagger} \\
L_{d}^{(0)} \frac{v}{m_{\mathrm{KKf}}} & \rightarrow Z_{L}^{d} L_{d}^{(0)} \frac{v}{m_{\mathrm{KKf}}} K_{L}^{u \dagger} \\
L_{u}^{(0)} \frac{v}{m_{\mathrm{KKf}}} & \rightarrow Z_{L}^{u} L_{u}^{(0)} \frac{v}{m_{\mathrm{KKf}}} K_{L}^{d \dagger} \\
\frac{v^{2}}{m_{\mathrm{KKf}}^{2}} & \rightarrow K_{L}^{u} \frac{v^{2}}{m_{\mathrm{KKf}}^{2}} K_{L}^{d \dagger}
\end{aligned}
$$

Thus, in diagrams with charged currents, we have two different flavor-mixing spurions, $L_{u}^{(0)}$ and $L_{d}^{(0)}$. In a totally general case with two flavor-mixing spurions, we could get $\bar{\theta}$ contributions with only two insertions of mixing matrices. However, the present case is far from generic, since there are no spurions other than $V_{\mathrm{CKM}}^{(0)}$ that are charged under both $Z_{L}^{u}$ and $Z_{L}^{d}$. Thus, in order to make an invariant from both $L_{u}^{(0)}$ and $L_{d}^{(0)}$, one must connect them with additional factors of $L_{u, d}^{(0)}$. We can therefore apply our earlier arguments about spurions charged under $Z_{L, R}^{u, d}$. The phase invariant $\Delta^{(4)}$ constructed from $L_{d}^{(0)}$ is approximately the same if we replace $L_{d}^{(0)}$ by $V_{\mathrm{CKM}}^{(0)}$ since most of the CKM matrix comes from the down-like quarks of our model. In particular, the largest new invariant that one can construct, even with charged currents, is still $\left(\frac{v}{m_{\mathrm{KKf}}}\right)^{7} \Delta^{(4)} \approx 10^{-16}$.

There is one last subtlety if the charged currents are Higgs (equivalently, longitudinal modes of the $W$ zero mode), since its coupling to KK modes in terms of the above spurions contains a large factor of $m_{\mathrm{KKf}} / v$. However, there is also no volume factor since the Higgs is IR localized and has no KK modes, so the overall largest contribution for instance from a charged Higgs and a KK gluon is of order $\delta \bar{\theta} \approx g_{4}^{2}\left(\frac{2 k \pi r_{c}}{(4 \pi)^{4}}\right)\left(\frac{v}{m_{\mathrm{KKf}}}\right)^{5} \Delta^{(4)} \lesssim 10^{-15}$.

\section{Precision electroweak constraints}

Here, for completeness, we review the constraints from precision electroweak measurements with a combination of bulk and brane fields. We will compute all constraints by matching our UV theory to an effective $4 \mathrm{~d}$ theory with only Standard Model fields. These constraints have been discussed previously in the literature, see e.g. [30-33]. The most highly constrained operators in the flavor-symmetric electroweak Lagrangian are

$$
\begin{aligned}
\mathcal{L}_{E W} \supset & \frac{z_{W B}}{\Lambda^{2}}\left(h^{\dagger} \sigma^{a} h\right) W_{\mu \nu}^{a} B^{\mu \nu}+\frac{z_{h}}{\Lambda^{2}}\left|h^{\dagger} D_{\mu} h\right|^{2} \\
& +\frac{z_{h l}^{s}}{\Lambda^{2}} i\left(h^{\dagger} D^{\mu} h\right)\left(\bar{l} \gamma_{\mu} l\right)+\frac{z_{h l}^{t}}{\Lambda^{2}} i\left(h^{\dagger} \sigma^{a} D^{\mu} h\right)\left(\bar{l} \gamma_{\mu} l\right) \\
& +\frac{z_{h q}^{s}}{\Lambda^{2}} i\left(h^{\dagger} D^{\mu} h\right)\left(\bar{q} \gamma_{\mu} q\right)+\frac{z_{h q}^{t}}{\Lambda^{2}} i\left(h^{\dagger} \sigma^{a} D^{\mu} h\right)\left(\bar{q} \gamma_{\mu} q\right) \\
& +\frac{z_{h u}}{\Lambda^{2}} i\left(h^{\dagger} D^{\mu} h\right)\left(\bar{u} \gamma_{\mu} u\right)+\frac{z_{h d}}{\Lambda^{2}} i\left(h^{\dagger} D^{\mu} h\right)\left(\bar{d} \gamma_{\mu} d\right)+\frac{z_{h e}}{\Lambda^{2}} i\left(h^{\dagger} D^{\mu} h\right)\left(\bar{e} \gamma_{\mu} e\right)
\end{aligned}
$$




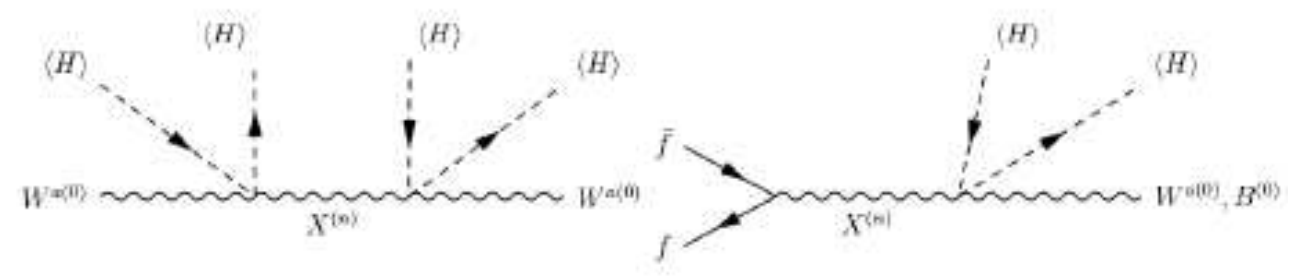

Figure 3: Diagrams that give contributions to electroweak observables at tree-level. The left diagram contributes to $T$ and the right contributes to fermion-Higgs operators. $X^{(n)}$ indicates KK modes of bulk gauge fields.

We present the constraints on the coefficients of these operators based on the $\chi^{2}$ comparison to the data performed in [31]. We consider only the 9 operators in equation $(\text { C.1 })^{9}$ the deviation from the $\chi^{2}$ for the standard model

$$
\Delta \chi^{2} \equiv \chi^{2}-\chi_{\mathrm{SM}}^{2}
$$

where $\chi_{\mathrm{SM}}^{2}$ is the $\chi^{2}$ value with all dimension- 6 operators set to zero. Choosing the 9 operator coefficients to minimize $\chi^{2}$ gives $\chi_{\min }^{2}=\chi_{\mathrm{SM}}^{2}-12.5$.

\section{C.1 Contributions from KK modes}

The contribution to fermion-Higgs operators occurs, as with most of the constrained higherdimensional operators, at tree-level. The diagrams that generate $z_{h \psi}$ are shown in figure 3 on the right. The contribution from hypercharge KK modes is ${ }^{10}$

$$
\begin{aligned}
& \frac{z_{h \psi}^{s}}{\Lambda^{2}} \approx \frac{1}{8} g^{\prime 2} z_{v}^{2} Y_{\psi} \times\left(\left(\begin{array}{c}
\frac{-k \pi r_{c}}{2}+\frac{1}{2} \psi \text { IR localized } \\
\frac{1}{4}-\frac{1}{4 k \pi r_{c}} \psi \text { UV localized }
\end{array}\right)\right) \\
& \frac{z_{h \psi}^{t}}{\Lambda^{2}} \approx \frac{1}{4} g^{2} z_{v}^{2} \times\left(\left(\begin{array}{c}
\frac{-k \pi r_{c}}{2}+\frac{1}{2} \psi \text { IR localized } \\
\frac{1}{4}-\frac{1}{4 k \pi r_{c}} \psi \text { UV localized }
\end{array}\right)\right)
\end{aligned}
$$

where $Y_{\psi}$ is the hypercharge of $\psi$ and $z_{v} \equiv \frac{1}{\mu_{\mathrm{TeV}}} \approx \frac{\sqrt{6}}{m_{K K g}}$ is the $z$-position of the IR brane. The contributions to $z_{h \psi}^{t}$ vanish when $\mathrm{SU}(2)_{L}$ is on the IR brane. Consider first the case where $\mathrm{SU}(2)_{L}$ is in the bulk. Then the constraints from the above operators depend on which fermions reside on the IR brane.

The leading contribution to $T$ is shown in figure f $_{\text {f }}$ on the left, and have been calculated before [35. ${ }^{11}$ The $\mathrm{U}(1)_{Y}$ KK mode contribution and the $\mathrm{SU}(2)_{L}$ KK mode contribution

\footnotetext{
${ }^{9}$ The constraints from 4 -fermion operators are negligible for the cases we discuss. Operators involving only quarks are weakly constrained and when $\mathrm{SU}(2)_{L}$ is in the bulk operators involving leptons may be made small by localizing the leptons sufficiently in the UV. When $\mathrm{SU}(2)_{L}$ is on the brane, the doublet leptons must be IR localized and thus the operators $\left(\bar{l} \gamma^{\mu} l\right)\left(\bar{l} \gamma_{\mu} l\right)$ and $\left(\bar{l} \gamma^{\mu} \sigma^{a} l\right)\left(\bar{l} \gamma_{\mu} \sigma^{a} l\right)$ are larger. However, in this case there are no KK modes of $W, Z$ bosons, and the effect of including constraints on these operators is small.

${ }^{10}$ See eq 5.2 in [35].

${ }^{11}$ Their equation 4.7 .
} 
give

$$
\begin{aligned}
& \Pi_{33}(0) \approx-\frac{v^{2}}{4} \frac{(k \pi r)\left(g^{2}+g^{\prime 2}\right)\left(v z_{v}\right)^{2}}{8}\left(1-\frac{1}{k \pi r_{c}}\right) \\
& \Pi_{11}(0) \approx-\frac{v^{2}}{4} \frac{(k \pi r)\left(g^{2}\right)\left(v z_{v}\right)^{2}}{8}\left(1-\frac{1}{k \pi r_{c}}\right)
\end{aligned}
$$

Recall

$$
\begin{aligned}
T & \approx \frac{-16 \pi\left(\Pi_{33}(0)-\Pi_{11}(0)\right)}{v^{2} e^{2}} \\
& \approx-\frac{16 \pi}{v^{2} g^{\prime 2} \cos ^{2} \theta_{W}}\left(\Pi_{33}(0)-\Pi_{11}(0)\right)
\end{aligned}
$$

It is related to the operator coefficient by

$$
\begin{aligned}
\frac{z_{h}}{\Lambda^{2}} & =-\frac{g^{\prime 2} \cos ^{2} \theta_{W}}{2 \pi v^{2}} T \\
& =-\frac{g^{\prime 2}}{4} k \pi r_{c} z_{v}^{2}\left(1-\frac{1}{k \pi r_{c}}\right)
\end{aligned}
$$

With $\mathrm{SU}(2)_{R}$ in the bulk, the leading order piece in the volume factor $\left(k \pi r_{c}\right)$ cancels. With $m_{h}=113$, we find, for $\Delta \chi^{2} \equiv \chi^{2}-\chi_{\mathrm{SM}}^{2}$,

\begin{tabular}{|c|c|c|c|c|}
\hline $\mathrm{SU}(2)_{L}$ & $\mathrm{SU}(2)_{R}$ & $L$ doublet & $m_{K K g, \Delta \chi^{2}=4.5}$ & $m_{K K g, \Delta \chi^{2}=9}$ \\
\hline In Bulk & In Bulk & In Bulk & 26.0 & 22.7 \\
\hline In Bulk & In Bulk & On Brane & 26.5 & 23.4 \\
\hline In Bulk & Not Present & In Bulk & 28.0 & 24.4 \\
\hline In Bulk & Not Present & On Brane & 22.4 & 20.0 \\
\hline On Brane & Not Present & On Brane & 15.6 & 14.2 \\
\hline
\end{tabular}

though as we have mentioned in order to address the strong $\mathrm{CP}$ problem we have $L$ doublets on the brane in our models. For 9 fit parameters, $\Delta \chi^{2}=\chi^{2}-\left(\chi_{\min , 9}^{2}+\right.$ $12.5)=4.5(9.0)$ for $95 \%(99 \%)$ confidence. Certain linear combinations of the fermionHiggs operators are effectively equivalent, via a field redefinition [32, 35], to contributions to $S$ and $T$. When the fermions are on the brane, this contribution to $S$ is effectively negative [30], whereas new physics usually gives a positive contribution to $S$. Therefore, it is not difficult, though somewhat ad hoc, to introduce new particles that alleviate these constraints. A less ad hoc option is to consider a heavy higgs, which increases $S$ at the price of decreasing $T$. Contours are shown in figure 1 for all doublets on the $\mathrm{TeV}$ brane, for $\mathrm{SU}(2)_{L}$ i) on the brane or ii) in the bulk, without $\mathrm{SU}(2)_{R}$ gauged. Minimizing $\chi^{2}$ over $m_{K K g}, m_{h}$ gives $\chi_{\min , 2}^{2}=\chi_{\mathrm{SM}}^{2}-2.13$ in the former case and $\chi_{\min , 2}^{2}=\chi_{\mathrm{SM}}^{2}-2.25$ in the latter case. In both cases, electroweak constraints force $m_{\text {gauge }}^{(1)} \gtrsim 15 \mathrm{TeV}$. 

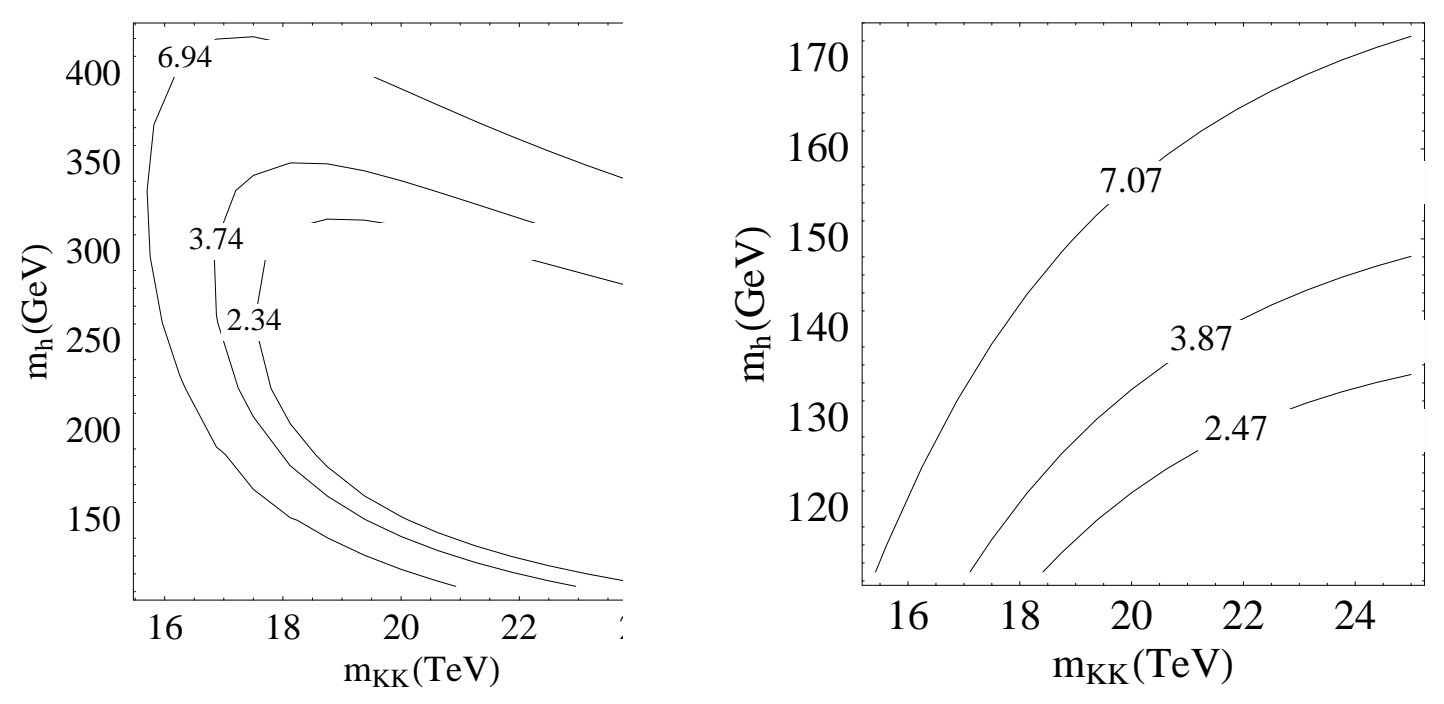

Figure 4: Plots of $\Delta \chi^{2}=\chi^{2}-\chi_{\mathrm{SM}}^{2}$ contours $(90 \%, 95 \%, 99 \%)$ when the doublets are all on the brane. The left(right) plot shows the case when $\mathrm{SU}(2)_{L}$ is in the bulk (on the $\mathrm{TeV}$ brane).

\section{Domain walls}

Since CP is a discrete symmetry that is spontaneously broken at the scale $E_{\mathrm{CP}}=\mu_{\mathrm{TeV}} t_{0}^{-1}$, domain walls will form as the universe cools and passes through a phase transition. It is therefore important that this phase transition happen only before inflation and not after. During inflation, the universe rapidly cools to the de Sitter temperature $T_{\mathrm{dS}}=E_{\mathrm{inf}}^{2} / M_{\mathrm{pl}}$, where $E_{\mathrm{inf}}=V_{\mathrm{inf}}^{1 / 4}$ is the energy scale of inflation. If $E_{\mathrm{CP}}>T_{\mathrm{dS}}$, then the phase transition will occur during inflation. There is also a danger that the universe will reheat above $E_{\mathrm{CP}}$, and then domain walls would form again after inflation, as the universe cooled from above to below $E_{\mathrm{CP}}$. In order to avoid this, it is necessary that the reheating temperature $T_{\mathrm{rh}}<E_{\mathrm{CP}}$. If reheating is efficient, then $T_{\mathrm{rh}}=E_{\mathrm{inf}}$, but in general the reheating temperature can be much lower if the efficiency is very small, i.e. $T_{\mathrm{rh}}=\epsilon_{\mathrm{eff}} E_{\mathrm{inf}}$. Both constraints are satisfied for $E_{\mathrm{CP}}=3 \mathrm{TeV}$ if for example $E_{\text {inf }} \approx 10^{11} \mathrm{GeV}$ and $\epsilon_{\mathrm{eff}} \approx 10^{-8}$.

\section{E. KK fermion mass matrix}

The complete mass matrix for the fermions involves the masses of the kk modes, which get contributions from the yukawa interactions. In order for $\bar{\theta}$ to truly vanish at tree-level, it is important that these mixing terms do not ruin the reality condition on the mass matrix determinant. In fact, it is easy to see that they do not, with or without doublets in the bulk. Consider first the case with doublets in the bulk. Then, being very explicit, before going to the yukawa eigenbasis, the mass matrix for the zero modes and the first two excited 
modes takes the following form:

$$
\left(\begin{array}{c}
u_{L}^{(0)} \\
u_{L}^{(1)} \\
u_{L}^{\prime(1)} \\
u_{L}^{(2)} \\
u_{L}^{\prime(2)}
\end{array}\right){ }^{T}\left(\begin{array}{ccccc}
v F^{q(0) \dagger} F^{u(0)} & 0 & v F^{q(0) \dagger} F^{u(1)} & 0 & v F^{q(0) \dagger} F^{u(2)} \\
v F^{q(1) \dagger} F^{u(0)} & M_{Q}^{(1)} & v F^{q(1) \dagger} F^{u(1)} & 0 & v F^{q(1) \dagger} F^{u(2)} \\
0 & 0 & M_{U}^{(1)} & 0 & 0 \\
v F^{q(2) \dagger} F^{u(0)} & 0 & v F^{q(2) \dagger} F^{u(1)} & M_{Q}^{(2)} & v F^{q(2) \dagger} F^{u(2)} \\
0 & 0 & 0 & 0 & M_{U}^{(2)}
\end{array}\right)\left(\begin{array}{c}
u_{R}^{(0)} \\
u_{R}^{\prime(1)} \\
u_{R}^{(1)} \\
u_{R}^{\prime(2)} \\
u_{R}^{(2)}
\end{array}\right)
$$

where $M_{U}^{(i)}$ denotes a diagonal, real mass matrix of the KK masses. This matrix is hermitian, thanks to the many empty entries, as long as $M_{U}^{(i)}, M_{Q}^{(i)}$, are hermitian and $F^{q(0) \dagger} F^{u(0)}$ has real determinant. Notice that $F^{q(i) \dagger} F^{u(j)}$ does not affect the determinant of this matrix be hermitian for any $(i, j)$ except for $(0,0)$.

When doublets are on the brane, the mass matrix is even simpler.

$$
\mathcal{L} \supset\left(\begin{array}{c}
u_{L}^{(0)} \\
u_{L}^{\prime(1)} \\
u_{L}^{\prime(2)}
\end{array}\right)^{T}\left(\begin{array}{ccc}
v F^{u(0)} & v F^{u(1)} & F^{u(2)} \\
0 & M_{U}^{(1)} & 0 \\
0 & 0 & M_{U}^{(2)}
\end{array}\right)\left(\begin{array}{c}
u_{R}^{(0)} \\
u_{R}^{(1)} \\
u_{R}^{(2)}
\end{array}\right)
$$

which clearly has real determinant.

\section{References}

[1] J.H. Christenson, J.W. Cronin, V.L. Fitch and R. Turlay, Evidence for the 2 pi decay of the $K(2)_{0}$ meson, Phys. Rev. Lett. 13 (1964) 138.

[2] A. Ceccucci, Z. Ligeti and Y. Sakai, The CKM quark-mixing matrix, in PARTicle DAtA Group collaboration, W.M. Yao et al., Review of particle physics, J. Phys. G 33 (2006) 1.

[3] G. 't Hooft, Symmetry breaking through Bell-Jackiw anomalies, Phys. Rev. Lett. 37 (1976) 8.

[4] M. Kamionkowski and J. March-Russell, Planck scale physics and the Peccei-Quinn mechanism, Phys. Lett. B 282 (1992) 137 hep-th/9202003.

[5] S.M. Barr and D. Seckel, Planck scale corrections to axion models, Phys. Rev. D 46 (1992) 539 .

[6] T.Banks, Report on progress in wormhole physics, Physicalia Mag. 12S2 (1990) 19.

[7] G. Dvali, A vacuum accumulation solution to the strong CP problem, Phys. Rev. D 74 (2006) 025019 hep-th/0510053.

[8] A. Pomarol, Gauge bosons in a five-dimensional theory with localized gravity, Phys. Lett. B 486 (2000) 153 hep-ph/9911294.

[9] H. Davoudiasl, J.L. Hewett and T.G. Rizzo, Bulk gauge fields in the Randall-Sundrum model, Phys. Lett. B 473 (2000) 43 hep-ph/9911262.

[10] S. Chang, J. Hisano, H. Nakano, N. Okada and M. Yamaguchi, Bulk standard model in the Randall-Sundrum background, Phys. Rev. D 62 (2000) 084025 hep-ph/9912498.

[11] Y. Grossman and M. Neubert, Neutrino masses and mixings in non-factorizable geometry, Phys. Lett. B 474 (2000) 361 hep-ph/9912408. 
[12] R. Harnik, G. Perez, M.D. Schwartz and Y. Shirman, Strong CP, flavor and twisted split fermions, JHEP 03 (2005) 068 hep-ph/0411132.

[13] H.-C. Cheng and D.E. Kaplan, Axions and a gauged Peccei-Quinn symmetry, hep-ph/0103346.

[14] A. Delgado, A. Pomarol and M. Quirós, Electroweak and flavor physics in extensions of the standard model with large extra dimensions, JHEP 01 (2000) 030 hep-ph/9911252.

[15] G.G. Raffelt, Astrophysical methods to constrain axions and other novel particle phenomena, Phys. Rept. 198 (1990) 1.

[16] L. Randall and M.D. Schwartz, Quantum field theory and unification in AdS5, JHEP 11 (2001) 003 hep-th/0108114.

[17] L. Randall and M.D. Schwartz, Unification and the hierarchy from AdS $S_{5}$, Phys. Rev. Lett. 88 (2002) 081801 hep-th/0108115.

[18] W.D. Goldberger and I.Z. Rothstein, Effective field theory and unification in AdS backgrounds, Phys. Rev. D 68 (2003) 125011 hep-th/0208060.

[19] K. Agashe, R. Contino and R. Sundrum, Top compositeness and precision unification, Phys. Rev. Lett. 95 (2005) 171804 hep-ph/0502222.

[20] K. Agashe, A. Delgado and R. Sundrum, Grand unification in RS1, Ann. Phys. (NY) 304 (2003) 145 hep-ph/0212028.

[21] H. Davoudiasl, J.L. Hewett and T.G. Rizzo, Experimental probes of localized gravity: on and off the wall, Phys. Rev. D 63 (2001) 075004 hep-ph/0006041].

[22] S.J. Huber, Flavor violation and warped geometry, Nucl. Phys. B 666 (2003) 269 hep-ph/0303183.

[23] Y. Nir, Probing new physics with flavor physics (and probing flavor physics with new physics), arXiv:0708.1872.

[24] Y. Nir, CP-violation: a new era, hep-ph/0109090.

[25] K. Agashe, G. Perez and A. Soni, Flavor structure of warped extra dimension models, Phys. Rev. D 71 (2005) 016002 hep-ph/0408134.

[26] A.E. Nelson, Naturally weak CP-violation, Phys. Lett. B 136 (1984) 387.

[27] S.M. Barr, Solving the strong CP problem without the Peccei-Quinn symmetry, Phys. Rev. Lett. 53 (1984) 329.

[28] G. Hiller and M. Schmaltz, Strong-weak CP hierarchy from non-renormalization theorems, Phys. Rev. D 65 (2002) 096009 hep-ph/0201251.

[29] J.R. Ellis and M.K. Gaillard, Strong and weak CP-violation, Nucl. Phys. B 150 (1979) 141.

[30] C. Csáki, J. Erlich and J. Terning, The effective lagrangian in the Randall-Sundrum model and electroweak physics, Phys. Rev. D 66 (2002) 064021 hep-ph/0203034.

[31] Z. Han and W. Skiba, Effective theory analysis of precision electroweak data, Phys. Rev. D 71 (2005) 075009 hep-ph/0412166.

[32] C. Grojean, W. Skiba and J. Terning, Disguising the oblique parameters, Phys. Rev. D 73 (2006) 075008 hep-ph/0602154]. 
[33] C.P. Burgess, S. Godfrey, H. Konig, D. London and I. Maksymyk, Model independent global constraints on new physics, Phys. Rev. D 49 (1994)6115 hep-ph/9312291.

[34] K. Agashe and R. Contino, The minimal composite Higgs model and electroweak precision tests, Nucl. Phys. B 742 (2006) 59 hep-ph/0510164.

[35] K. Agashe, A. Delgado, M.J. May and R. Sundrum, RS1, custodial isospin and precision tests, JHEP 08 (2003) 050 hep-ph/0308036.

[36] C.G. Callan Jr. and J.A. Harvey, Anomalies and fermion zero modes on strings and domain walls, Nucl. Phys. B 250 (1985) 427.

[37] N. Arkani-Hamed, A.G. Cohen and H. Georgi, Anomalies on orbifolds, Phys. Lett. B 516 (2001) 395 hep-th/0103135. 